\title{
Guide to the Atlas Software: Computational Representation Theory of Real Reductive Groups
}

\author{
Jeffrey Adams \\ April 25, 2008 \\ In memory of Fokko du Cloux
}

\section{Introduction}

The Atlas of Lie Groups and Representations is a project in the representation theory of real reductive groups. The main goal of the atlas computer software, currently under development, is to compute the unitary dual of any real reductive Lie group $G$. As a step in this direction it currently computes the admissible representations of $G$.

The underlying mathematics of the software is described in Algorithms for Representation Theory of Real Reductive Groups [1]. See Sections 1 and 2 of [1] for an overview of the algorithm. This paper is a complement, and consists of a guide to the software illustrated by numerous examples.

The software is currently in an early stage of development (version 0.3 as of April 2008). It is available from the Atlas web site www.liegroups.org.

The help command in the atlas software is a another useful source of information. Also see the software section of www.liegroups.org, including examples and Tables of Structure and Representation Theory. We plan to publish a manual for the software at the time version 1.0 is released.

Marc van Leeuwen is currently working on the interpreter, which provides simpler and more powerful input and output methods for the software. This is included in the current software distribution; see the makefile and the source/interpreter directory. We do not discuss this here since it is in a 
state of flux. The input methods described in this paper will be available indefinitely. The interpreter will provide additional output routines, but there should only be minor changes to the output described here.

The atlas software was written by Fokko du Cloux. Alfred Noel has also contributed to it. In addition to writing the interpreter, Marc van Leeuwen has made substantial changes to the software, and since version 0.3 has been in charge of software development.

This paper grew out of lectures at the conference in honor of Dragan Milicic and Bill Casselman at Snowbird, July 2006. Bill had a catalytic effect on the Atlas project, which got started at a conference he organized in Montreal in 2002.

\subsection{How to read this paper}

You should start by downloading the atlas software from the Atlas web site www.liegroups.org and installing it. Currently it runs under unix, including Solaris and linux, Mac OSX, and Windows. You should also have the paper Algorithms for Representation Theory of Real Reductive Groups handy.

A good approach to learning the software and the Algorithms paper is go through this paper, and do the examples using the software. Each section begins with a brief summary of the relevant material from Algorithms. We being very simply, and build up to more complicated examples by the end. We assume some familiarity with the theory of real reductive groups, for example see Chapters 1-8 of [2].

\section{Defining basic data}

The starting point of the algorithm is a complex reductive algebraic group $G$ together with an inner class of real forms of $G$. The latter is determined by an involution $\gamma \in \operatorname{Out}(G)$, the group of outer automorphisms of $G$. We refer to $(G, \gamma)$ as basic data $[1$, Section 4$]$.

We define a semidirect product $G^{\Gamma}=G \rtimes \Gamma$, where $\Gamma=\operatorname{Gal}(\mathbb{C} / \mathbb{R})=$ $\{1, \sigma\}$. We let $\sigma$ act on $G$ by a "distinguished" involution $\tau \in \operatorname{Aut}(G)$ mapping to $\gamma \in \operatorname{Out}(G)$, via the map $\operatorname{Aut}(G) \rightarrow \operatorname{Out}(G)$. (A distinguished involution is one which fixes a splitting datum $\left(B, H,\left\{X_{\alpha}\right\}\right)$; it is the "most 
compact" involution in this inner class.) Write $G^{\Gamma}=\langle G, \delta\rangle$ where $\delta=1 \times \sigma$. See [1, Section 5].

A real form of $G$ in this inner class is a conjugacy class of involutions $\theta \in \operatorname{Aut}(G)$ mapping to $\gamma \in \operatorname{Out}(G)$. If $\theta$ is an involution of $G$ let $\sigma$ be an antiholomorphic involution of $G$ commuting with $\theta$. Then $G(\mathbb{R})=G^{\sigma}$ is a real group in the usual sense, and $G(\mathbb{R})^{\theta}$ is a maximal compact subgroup of $G(\mathbb{R})$. See $[1$, Section 5$]$.

The reader should keep in mind the basic example of $\gamma=1$, so $G^{\Gamma}=$ $G \times \Gamma$. This is known as the compact or equal rank inner class; it contains the compact real form of $G$, and all groups in this inner class contain a compact Cartan subgroup.

The first step in using the atlas software is to define basic data $(G, \gamma)$. This proceeds in three steps:

(1) Define a complex reductive Lie algebra $\mathfrak{g}$, and let $G^{*}$ be the product of a complex torus and a simply connected, semisimple complex group with Lie algebra $\mathfrak{g}$;

(2) Choose a finite subgroup $A$ of $Z\left(G^{*}\right)$, and set $G=G^{*} / A$;

(3) Choose an inner class of real forms of $G$.

All three steps are accomplished by the type command. We break this up into three steps.

\subsection{Defining $\mathfrak{g}$ and $G^{*}$}

A complex reductive Lie algebra is given by a list of types $A \_n, B \_n, \ldots$, E_8, T_n, where $\mathrm{T} \_n$ is the abelian Lie algebra $\mathbb{C}^{n}$. In response to the type command, the software asks for the Lie type:. The user then enters such a list, with terms separated by a period. The order is irrelevant here, although it plays a role in steps (2) and (3). The entry T_2 is the same as T_1.T_1.

This defines the complex reductive Lie algebra $\mathfrak{g}$, and group $G^{*}$. Here are some simple examples.

Example 2.1 We start with $S L(2, \mathbb{C})$ :

main: type

Lie type: A1 
Here is $S L(2, \mathbb{C}) \times S L(2, \mathbb{C})$ :

main: type

Lie type: A1.A1

Here is $S L(2, \mathbb{C}) \times \mathbb{C}^{\times}$:

main: type

Lie type: A1.T1

and finally $S L(2, \mathbb{C})^{2} \times \operatorname{Spin}(5, \mathbb{C}) \times S p(4, \mathbb{C}) \times S p(6, \mathbb{C}) \times \mathbb{C}^{\times 4}$ :

main: type

Lie type: A1.T1.B2.C2.T1.C3.T2.A1

\subsection{Defining a complex group $G$}

The second step is to pick a finite subgroup of the center $Z^{*}$ of $G^{*}$.

The center of each simple factor of $G$ is a finite cyclic group except in type $D_{2 n}$ in which case it is $\mathbb{Z}_{2} \times \mathbb{Z}_{2}$. The finite cyclic group of order $n$ is denoted $\mathrm{Z} / \mathrm{n}$, and is viewed as the group

$$
\frac{1}{n} \mathbb{Z} / \mathbb{Z}=\left\{\frac{0}{n}, \frac{1}{n}, \ldots, \frac{n-1}{n}\right\} .
$$

The elements of finite order in $\mathbb{C}^{\times}$are isomorphic to $\mathbb{Q} / \mathbb{Z}$, and an element of this group is given by an element of $\mathbb{Q}$.

Once the user has given the Lie type, the software prompts the user for a finite subgroup of $Z^{*}$, generated by a set of elements of $Z^{*}$. Each such element is given by a list of fractions, one for each term in the Lie type (two for $D_{2 n}$ ), separated by commas. Each element is given on a single line; the empty line terminates this aspect of the input. For example simply typing return in response to this prompt takes $A=1$ and $G=G^{*}$. Typing sc has the same effect. Typing ad gives the adjoint group (actually a torus times the adjoint group of the derived group of $G^{*}$ ).

Example 2.2 Here is the group $S L(2, \mathbb{C})$ :

main: type

Lie type: A1

elements of finite order in the center of the simply connected group: 
$\mathrm{Z} / 2$

enter kernel generators, one per line

(ad for adjoint, ? to abort):

sc

enter inner class(es):

Entering a carriage return alone also gives $S L(2, \mathbb{C})$.

Example 2.3 To define $P S L(2, \mathbb{C})$ take $A$ to be the center of $S L(2, \mathbb{C})$, which is generated by the element of order 2 :

Lie type: A1

elements of finite order in the center of the simply connected group:

$\mathrm{Z} / 2$

enter kernel generators, one per line

(ad for adjoint, ? to abort):

$1 / 2$

enter inner class(es):

Entering ad instead of $1 / 2$ has the same effect.

Example 2.4 Here is $S O(10, \mathbb{C})$, which is the quotient of $\operatorname{Spin}(10, \mathbb{C})$ by the element of $Z^{*}$ of order 2 :

main: type

Lie type: D5

elements of finite order in the center of the simply connected group:

$\mathrm{Z} / 4$

enter kernel generators, one per line

(ad for adjoint, ? to abort):

$2 / 4$

Often a reductive group is a quotient of $G^{*}$ by a diagonal subgroup.

Example 2.5 Here is $G L(2, \mathbb{C}) \simeq S L(2, \mathbb{C}) \times \mathbb{C}^{*} /\{(-I,-1)\}$ : 
main: type

Lie type: A1.T1

elements of finite order in the center of the simply connected group:

$\mathrm{Z} / 2 . \mathrm{Q} / \mathrm{Z}$

enter kernel generators, one per line

(ad for adjoint, ? to abort):

$1 / 2,1 / 2$

The center of $\operatorname{Spin}(4 n, \mathbb{C})$ is not cyclic, and therefore requires two terms.

Example 2.6 Here is $S O(8, \mathbb{C}) \simeq \operatorname{Spin}(8, \mathbb{C}) / A$ where $A$ is the diagonal subgroup of $Z^{*}=\mathbb{Z}_{2} \times \mathbb{Z}_{2}$ :

main: type

Lie type: D4

elements of finite order in the center of the simply connected group:

$\mathrm{Z} / 2 . \mathrm{Z} / 2$

enter kernel generators, one per line

(ad for adjoint, ? to abort):

$1 / 2,1 / 2$

Example 2.7 We can also take the quotient of $\operatorname{Spin}(8, \mathbb{C})$ by a non-diagonal

$\mathbb{Z}_{2}$ subgroup:

main: type

Lie type: D8

elements of finite order in the center of the simply connected group:

$\mathrm{Z} / 2 . \mathrm{Z} / 2$

enter kernel generators, one per line

(ad for adjoint, ? to abort):

$1 / 2,0 / 2$

This is not isomorphic to $S O(8, \mathbb{C})$.

This defines the group $G=G^{*} / A$ and completes step 2 . 


\subsection{Defining an inner class of real forms}

We next define an inner class of real forms. Recall this is determined by an involution in $\operatorname{Out}(G)$.

The trivial element of $\operatorname{Out}(G)$ corresponds to the inner class of real forms containing a compact Cartan subgroup. This is the compact inner class, and is denoted c, and also e for equal rank. In particular if $G$ has a torus factor its real points are $S^{1} \times \cdots \times S^{1}$.

Another natural inner class is that of the split real form This class is denoted $\mathbf{s}$; for a torus the real points are $\mathbb{R}^{\times} \times \cdots \times \mathbb{R}^{\times}$. In many cases the classes c and $\mathbf{s}$ are the same, for example if $\operatorname{Out}(G)=1$.

Now suppose $G=G_{1} \times G_{1}$. The outer automorphism switching the two factors corresponds to the inner class of the real form $G_{1}(\mathbb{C})$, viewed as a real group. This class is denoted $\mathrm{C}$; in the case of a torus this gives $\mathbb{C}^{\times} \times \cdots \times \mathbb{C}^{\times}$ (viewed as a real group).

If $G$ is simple, then except in type $D_{2 n}$ the classes c, s exhaust every inner class. In type $D_{2 n}$ the classes c and s coincide, and there is another inner class denoted $\mathrm{u}$, for unequal rank. See example 2.14. This class is allowed in any type for which the Dynkin diagram has a non-trivial automorphism.

Now suppose $G=G^{*}$. An inner class of real forms of $G$ is specified by choosing c,e, s, u for each simple or $T^{1}$ factor, or $\mathrm{C}$ for each pair of identical (simple or $T^{1}$ ) factors, or factor of type $T^{2 n}$. In general an inner class of real forms of $G$ is given by an allowed inner class of real forms of $G^{*}$ : the involutions in this inner class must factor to $G$.

To summarize, to specify an inner class of real forms of $G$, give a list of choices for each simple or torus factor, or pair of identical entries in the case of $C$ :

- c: compact

- e: equal rank (same as c)

- s: split

- u: unequal rank

- C: complex (for an identical pair of entries). 
The order of the choices corresponds to the order in which the simple and torus factors of $G^{*}$ were specified. If $G \neq G^{*}$ some choices may not be allowed.

Here are some examples. Getting slightly ahead of ourself, the showrealforms command lists the real forms in the given inner class. In the terminology of atlas these are weak real forms; see Sections 3 and 4. Also note that the first three inputs to the type command can be entered on a single line, provided the second input is ad or sc.

Example 2.8 The group $S L(2, \mathbb{C})$ has two real forms $S L(2, \mathbb{R})$ and $S U(2)$, both of which are in the same inner class. Thus $\mathrm{c}=\mathrm{e}=\mathrm{s}$ in this case.

empty: showrealforms

Lie type: A1 sc s

(weak) real forms are:

$0: \mathrm{su}(2)$

1: $\operatorname{sl}(2, R)$

This example also illustrates a general principle of the software: entering a command such as showrealforms which requires other input is allowed; the system will prompt the user for the missing information.

Example 2.9 The smallest simple group with two inner classes is type $A_{2}$. Here is the split inner class, with only one real form:

empty: showrealforms

Lie type: A2 sc s

(weak) real forms are:

$0: \operatorname{sl}(3, R)$

Here is the compact inner class, with two real forms:

empty: showrealforms

Lie type: A2 sc c

(weak) real forms are:

$0: \mathrm{su}(3)$

1: $\operatorname{su}(2,1)$

Example 2.10 Here is $S L(2, \mathbb{C})$ viewed as a real group: 
empty: showrealforms

Lie type: A2.A2 sc C

(weak) real forms are:

$0: \operatorname{sl}(3, \mathrm{C})$

Example 2.11 If the group is a product specify the inner class as a list. For example here is the inner class of $S U(3) \times S L(3, \mathbb{R})$ :

empty: showrealforms

Lie type: A2.A2 sc cs

(weak) real forms are:

$0: \operatorname{su}(3) . \operatorname{sl}(3, R)$

1: $\operatorname{su}(2,1) \cdot \operatorname{sl}(3, R)$

(The sc means simply connected, and cs means compact $\times$ split).

Example 2.12 Here is the inner class of $G L(2, \mathbb{R})$ :

empty: showrealforms

Lie type: A1.T1

elements of finite order in the center of the simply connected group:

$\mathrm{Z} / 2 . \mathrm{Q} / \mathrm{Z}$

enter kernel generators, one per line

(ad for adjoint, ? to abort):

$1 / 2,1 / 2$

enter inner class(es): ss

(weak) real forms are:

$0: \operatorname{su}(2) \cdot g l(1, R)$

1: $\operatorname{sl}(2, R) \cdot g l(1, R)$

Group 1 is $G L(2, \mathbb{R})$ (group 0 is the multiplicative group of the quaternions).

Example 2.13 On the other hand here is the inner class of $U(1,1)$ :

main: showrealforms

Lie type: A1.T1

elements of finite order in the center of the simply connected group:

$\mathrm{Z} / 2 . \mathrm{Q} / \mathrm{Z}$

enter kernel generators, one per line 
(ad for adjoint, ? to abort):

$1 / 2,1 / 2$

enter inner class(es): cc

(weak) real forms are:

$0: \mathrm{su}(2) \cdot \mathrm{u}(1)$

1: $\operatorname{sl}(2, R) \cdot u(1)$

Groups 0 and 1 are $U(2)$ and $U(1,1)$, respectively.

Example 2.14 The group $S O(12, \mathbb{C})$ has the following real forms, in two inner classes. One inner class consists of the groups $S O(12,0), S(10,2)$, $S O(8,4), S O(6,6)$ and $S O^{*}(12)$. This is both the compact inner class (it contains $S O(12,0)$ ) and split (it contains $S O(6,6)$ ), and is therefore the class c=e=s. There is another inner class consisting of $S O(11,1), S O(9,3)$ and $S O(7,5)$. This is the inner class u of unequal rank. This is an example where $\mathrm{u}$ is needed.

Here is the compact and split inner class:

empty: showrealforms

Lie type: D6

elements of finite order in the center of the simply connected group: $\mathrm{Z} / 2 . \mathrm{Z} / 2$

enter kernel generators, one per line

(ad for adjoint, ? to abort):

$1 / 2,1 / 2$

enter inner class(es): e

(weak) real forms are:

0 : so(12)

1: $\operatorname{so}(10,2)$

2: $\operatorname{so} *(12)[1,0]$

3: $\operatorname{so} *(12)[0,1]$

4: so $(8,4)$

5: so $(6,6)$

For an explanation of the two version of so*(12) see Example 3.3.

The same input with $\mathrm{u}$ in place of e gives the unequal rank inner class: 


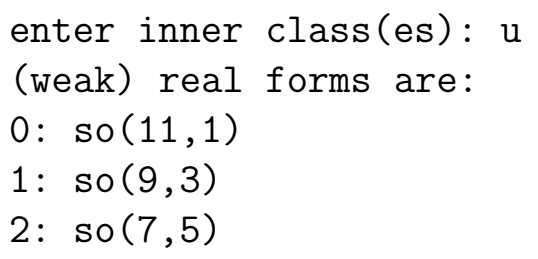

Example 2.15 Here is an example in which the inner class for $G^{*}$ is not defined for $G$ :

main: type

Lie type: A1.A1

elements of finite order in the center of the simply connected group: $\mathrm{Z} / 2 . \mathrm{Z} / 2$

enter kernel generators, one per line

(ad for adjoint, ? to abort):

$1 / 2,0 / 2$

enter inner class(es): C

sorry, that inner class is not compatible with the weight lattice

What this means is: the automorphism which switches the two factors in $G^{*}=S L(2, C) \times S L(2, \mathbb{C})$ does not preserve $A$, and so does not factor to $G=P S L(2, \mathbb{C}) \times S L(2, \mathbb{C})$.

Example 2.16 A more subtle example is the non-diagonal quotient of $\operatorname{Spin}(8, \mathbb{C})$ of Example 2.7:

main: type

Lie type: D4

elements of finite order in the center of the simply connected group: $\mathrm{Z} / 2 . \mathrm{Z} / 2$

enter kernel generators, one per line

(ad for adjoint, ? to abort):

$1 / 2,0 / 2$

enter inner class(es): u

sorry, that inner class is not compatible with the weight lattice 


\section{Defining a real group}

We suppose the user has completed the type command, which defines $G$ and an inner class of real forms. We next specify a particular real form of $G$ in the inner class. We discuss strong real forms in the next section.

The command showrealforms, discussed in the preceding section, gives a list of the real forms of $\mathfrak{g}$ in the given inner class; the command realform gives the same list, and the user can choose the real form from the list.

The real forms of the classical Lie algebras are given in the usual notation. Some examples are $s l(4, R), \operatorname{su}(3,1), \operatorname{su}(4), \mathrm{sl}(4, H), \operatorname{sp}(3,2), \mathrm{so}(3,2)$, and so*(10). For a torus the real form is specified by $g l(1, R), u(1)$ or $\mathrm{gl}(1, \mathrm{C})$.

For each exceptional group the real form is specified by specifying the type of the maximal compact subgroup, except that the split real form is denoted R. For example the real forms of $E_{7}$ are e7, e7 (e6.u(1)), e7 (so(12).su(2)) and $\mathrm{e} 7(\mathrm{R})$; otherwise known as compact, Hermitian, quaternionic and split, respectively.

Example 3.1 Here is the group $S L(2, \mathbb{R})$ :

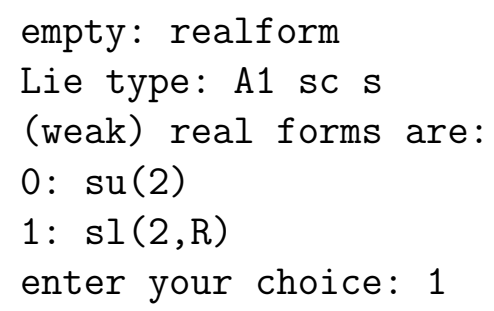

Example 3.2 Here is $P S L(2, \mathbb{R}) \simeq P G L(2, \mathbb{R}) \simeq S O(2,1)$.

main: type

Lie type: A1 ad $\mathrm{s}$

main: realform

(weak) real forms are:

$0: \mathrm{su}(2)$

1: $\operatorname{sl}(2, R)$

enter your choice: 1

Note that the real forms listed don't depend on the fact that the complex group is $P S L(2, \mathbb{C})$ here instead of $S L(2, \mathbb{C})$; the real form is determined by a real form of $\mathfrak{g}$. 
Example 3.3 The equal rank forms of $S O(12, \mathbb{C})$ were given in Example 2.14:

$0:$ so(12)

1: $\operatorname{so}(10,2)$

2: $\operatorname{so} *(12)[1,0]$

3: $\operatorname{so} *(12)[0,1]$

4: so $(8,4)$

5: so $(6,6)$

The two copies of so* (12) illustrate a technical point. We say two real forms are equivalent if they are conjugate by $G$. See $[1$, Section 5$]$. In the literature equivalence is defined to be conjugacy by $\operatorname{Aut}(G)$. If $G$ is simple the two notions agree in most cases.

There are two real forms (in our sense) of $S O(12, \mathbb{C})$ corresponding to the real group $S O^{*}(12)$. In other words there are two isomorphic subgroups of $S O(12, \mathbb{C})$, which are not conjugate to each other. They are related by an outer automorphism of $S O(12, \mathbb{C}$ ). (In the Kac classification of real forms each is labelled by a 2 on one of the branches of the fork in the Dynkin diagram). These two groups are denoted $S O^{*}(12)[1,0]$ and $S O^{*}(12)[0,1]$. The fact that there are two copies of $S O^{*}(12)$ manifests itself in the representation theory of $S O(p, q)$. See Example 8.8.

In fact we can even see this distinction in terms of the structure theory of the groups themselves. If $G=\operatorname{Spin}(12, \mathbb{C}), S O(12, \mathbb{C})$ or $P S O(12, \mathbb{C})$ the two real groups locally isomorphic to $S O^{*}(12)$ are isomorphic, and interchanged by an outer automorphism of $G$. However in the non-diagonal quotient of $\operatorname{Spin}(12, \mathbb{C})$ this fails, as is illustrated in the next example.

Example 3.4 Here are two versions of $S O^{*}(12)$ in the non-diagonal quotient of $\operatorname{Spin}(12, \mathbb{C})$ :

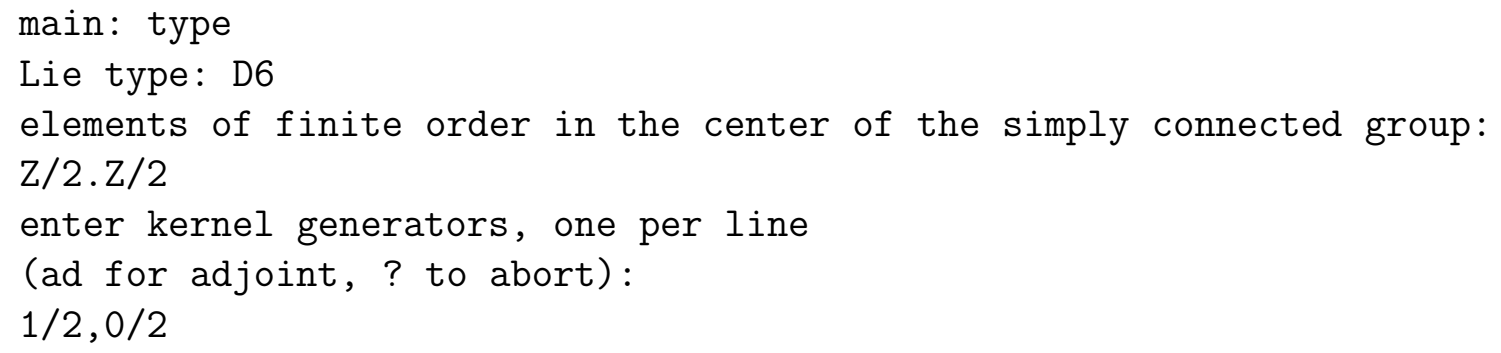




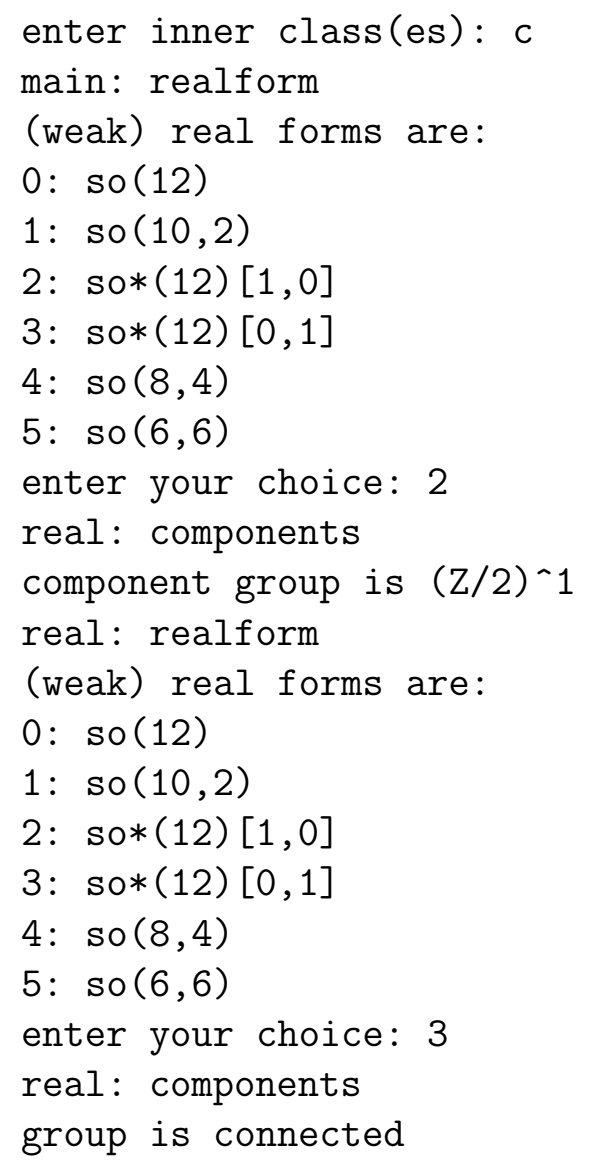

These two groups are not isomorphic. This illustrates the command components, which gives the component group of the real group (an elementary abelian two-group).

In $D_{4}$ the situation is even more interesting. Let $G=\operatorname{Spin}(8, \mathbb{C})$ and consider the three real forms, $\operatorname{Spin}(6,2)$ and the two versions of $\operatorname{Spin}^{*}(8)$. In fact these are isomorphic; $\operatorname{Spin}(6,2) \simeq \operatorname{Spin}^{*}(8)$, and these three groups are interchanged by the outer automorphism group of $\operatorname{Spin}(8, \mathbb{C})$, which is $S_{3}$. Similar statements hold in $P S O(8, \mathbb{C})$. We leave it to the reader to analyze real forms of $S O(8, \mathbb{C})$ and the non-diagonal quotient of $\operatorname{Spin}(8, \mathbb{C})$, in which this symmetry is broken in different ways. See Examples 2.6 and 2.7.

In general in $S O\left(4 n, \mathbb{C}\right.$ ) (type $\left.D_{2 n}\right)$ there are two non-conjugate copies of $S O^{*}(4 n)$. However in $S O(4 n+2, \mathbb{C})\left(\right.$ type $\left.D_{2 n+1}\right)$ all copies of $S O^{*}(4 n+2)$ are in fact conjugate by $S O(2 n+2, \mathbb{C})$. This is illustrated by the next example. 
Example 3.5 Here are the equal rank real forms of $S O(10, \mathbb{C})$ :

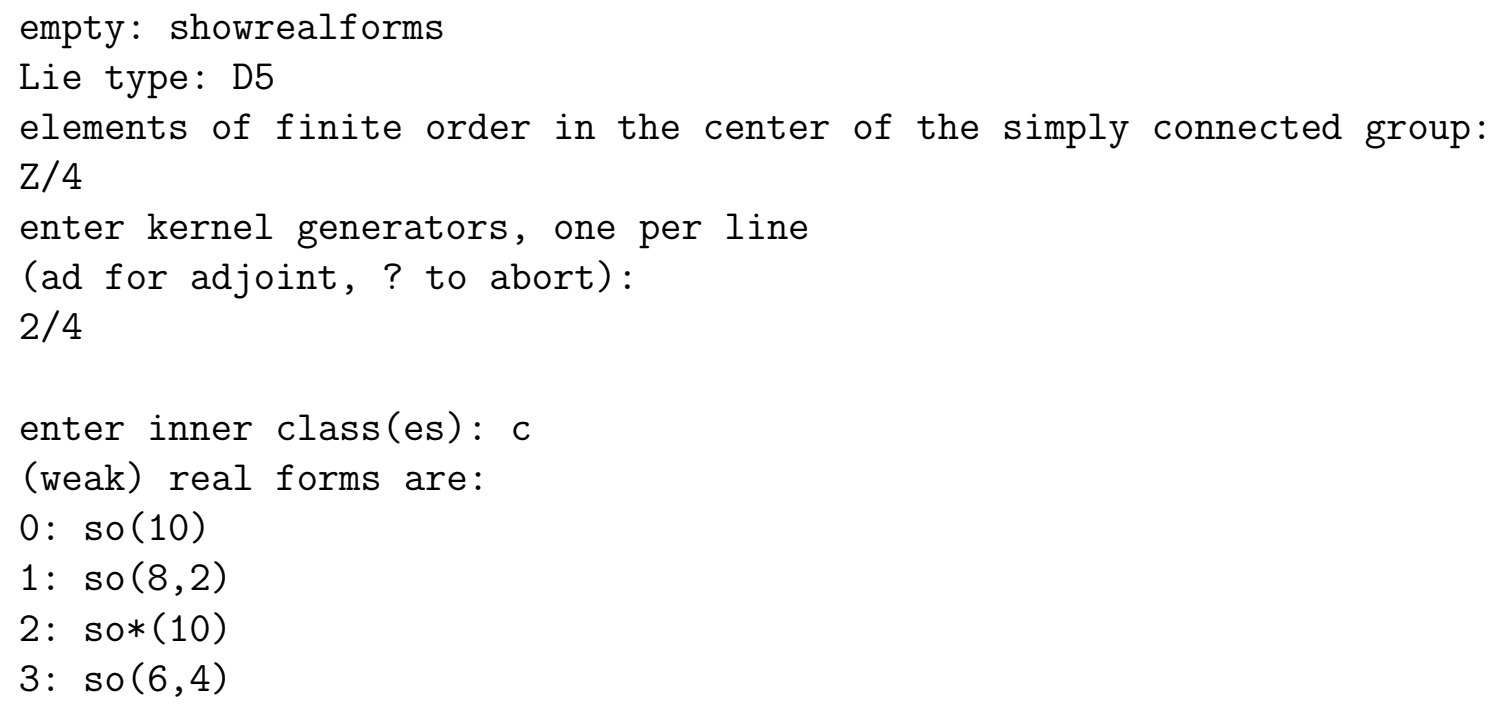

Note that there is only one real form $S O^{*}(10)$.

\section{The Spaces $\mathcal{X}$ and $\mathcal{X}^{r}$}

Fix basic data $(G, \gamma)$ as in Section 2 .

By a strong involution for $(G, \gamma)$ we mean an element $\xi \in G^{\Gamma} \backslash G$ satisfying $\xi^{2} \in Z(Z=Z(G)$ is the center of $G)$. Let $\mathcal{I}$ be the set of strong involutions. A strong real form is an equivalence class of strong involutions (equivalence is by conjugacy by $G$ ). If $\xi$ is a strong involution then $\theta_{\xi}=\operatorname{int}(\xi)$ is an involution of $G$ in the inner class of $\gamma$, and this gives a surjective map from strong real forms to real forms in this inner class. If $G$ is adjoint this map is bijective, but not otherwise. Let $K_{\xi}=G^{\theta_{\xi}}$; this is the complexified maximal compact subgroup of the corresponding real group $G(\mathbb{R})$. See Section 2 .

Recall (Section 2) $\delta=1 \times \sigma$ is the distinguished element of $G^{\Gamma}$. We fix once and for all a Cartan subgroup $H$ of $G$ stable by $\operatorname{int}(\delta)$. Define

$$
\begin{aligned}
\widetilde{\mathcal{X}} & =\left\{g \in \operatorname{Norm}_{G^{\Gamma} \backslash G}(H) \mid g^{2} \in Z\right\} \\
\mathcal{X} & =\widetilde{\mathcal{X}} / H
\end{aligned}
$$

(the quotient is by the conjugation action of $H$ ). See [1, Section 9]. The space $\mathcal{X}$ is our main combinatorial object, known as the (one sided) parameter space. 
Mathematically the space $\mathcal{X}$ is a natural object. From the point of view of computations it has the disadvantage that (if $G$ is not semisimple) it may be infinite, and the fibers of the map from strong real forms to real forms may be infinite. The software works exclusively with the reduced parameter space $\mathcal{X}^{r}$ which we now describe. See [1, Section 13].

Let $\theta=\operatorname{int}(\delta)$ and choose a set $Z^{r} \subset Z$ of representatives of

$$
\{z \in Z \mid \theta(z)=z\} /\{z \theta(z) \mid z \in Z\}
$$

Define reduced versions of $\mathcal{I}, \widetilde{\mathcal{X}}$ and $\mathcal{X}$ :

$$
\begin{aligned}
\mathcal{I}^{r} & =\left\{g \in G^{\Gamma} \backslash G \mid g^{2} \in Z^{r}\right\} \\
\widetilde{\mathcal{X}}^{r} & =\left\{g \in \operatorname{Norm}_{G^{\Gamma} \backslash G}(H) \mid g^{2} \in Z^{r}\right\}, \mathcal{X}^{r}=\widetilde{\mathcal{X}}^{r} / H .
\end{aligned}
$$

Henceforth we define a strong real form in this revised sense: $\mathcal{I}^{r}$ is the space of strong real forms, with equivalence given by conjugacy by $G$ as before. The map from strong real forms to real forms is still surjective. The fibers of this map are finite, and $\mathcal{X}^{r}$ is a finite set.

Choose a set $\left\{\xi_{i} \mid 1 \leq i \leq n\right\} \subset \widetilde{\mathcal{X}}$ of representatives of strong real forms (in the sense of the reduced parameter space). That is

$$
\left\{\xi_{1}, \ldots, \xi_{n}\right\} \stackrel{1-1}{\longleftrightarrow} \mathcal{I}^{r} / G .
$$

For each $i$ let $x_{i}$ be the image of $\xi_{i}$ in $\mathcal{X}^{r}, \theta_{i}=\operatorname{int}\left(\xi_{i}\right)$ and $K_{i}=G^{\theta_{i}}$. See $[1$, $5.15]$.

Let $W^{\Gamma}=\operatorname{Norm}_{G^{\Gamma}}(H) / H$ and

$$
\mathcal{I}_{W}=\left\{\tau \in W^{\Gamma} \backslash W \mid \tau^{2}=1\right\} .
$$

We continue to write $\delta$ for the image of $\delta \in G^{\Gamma}$ in $W^{\Gamma}$, and $\theta$ for the involution $\operatorname{int}(\delta)$ of $W$. Then $W^{\Gamma}=\langle W, \delta\rangle$. By a twisted involution in $W$ we mean an element $w \in W$ satisfying $w \theta(w)=1$, and we say two such elements $w, w^{\prime}$ are twisted-conjugate if $w^{\prime}=y w \theta\left(y^{-1}\right)$ for some $y \in W$. The map $w \rightarrow w \delta$ is a bijection between the space of twisted involutions and $\mathcal{I}_{W}$, taking twistedconjugacy to ordinary conjugacy by $W$. We refer to $\mathcal{I}_{W}$ as the space of twisted involutions, and pass back and forth between the two notions. See $[1,9.14]$.

There is a natural surjective map $p: \mathcal{X}^{r} \rightarrow \mathcal{I}_{W}[1$, Lemma 9.12]. The fiber over an element $\tau \in \mathcal{I}_{W}$ is denoted $\mathcal{X}_{\tau}^{r}$. 
The group $\operatorname{Norm}_{G}(H)$ acts by conjugation on $\widetilde{\mathcal{X}}^{r}$, and this factors to an action of $W$ on $\mathcal{X}^{r}$. Because of its relationship to the cross action of [3, Definition 8.3.1] we call this the cross action of $W$, and denote it $w \times x$. This action is equivariant for $p$ and the conjugation action of $W$ on $\mathcal{I}_{W}$ : $p(w \times x)=w p(x) w^{-1}$.

\section{Cartan subgroups}

Fix basic data $(G, \gamma)$. Let $\theta_{q s}$ be a quasisplit involution in this inner class and let $K_{q s}=G^{\theta_{q s}}$. Thus $K_{q s}$ is the complexified maximal compact subgroup of the quasisplit form $G_{q s}(\mathbb{R})$ of $G$. The conjugacy classes of Cartan subgroups of $G_{q s}(\mathbb{R})$, equivalently the $K_{q s}$-conjugacy classes of $\theta_{q s}$-stable Cartan subgroups of $G$, are in natural bijection with $\mathcal{I}_{W} / W$, conjugacy classes of twisted involutions in $W$. The conjugacy classes of Cartan subgroups for any real form of $G$ are a subset of those for the quasisplit real form. See [1, Propositions 12.9 and 12.12].

The cartan command gives a list of Cartan subgroups for a given inner form. For each Cartan subgroup it gives the following information. See the help file for the cartan command for more details.

First it gives the structure of the Cartan subgroup as a real torus: $H(\mathbb{R})$ is isomorphic to $\left(\mathbb{R}^{\times}\right)^{a} \times\left(S^{1}\right)^{b} \times\left(\mathbb{C}^{\times}\right)^{c}$ for integers $(a, b, c)$; these are the split, compact and complex entries in the output of cartan.

Each conjugacy class of twisted involutions contains a unique canonical representative $\tau=w \delta$. The next line of output is a reduced expression for $w$.

The first entry on the next line is the number of twisted involutions in this conjugacy class. For an explanation of fiber rank see and \#X_r see Section 9 .

Associated to any Cartan subgroup are the sets of real and imaginary roots (see Section 7), each of which is a root system. These are given by imaginary root system and real root system, respectively. The complex factor line in the output has to do with the Weyl group; see Section 6 .

The lines beginning real form... give information about $\mathcal{X}_{\tau}^{r}$. For now we observe only that for a given Cartan subgroup only the real forms which contain this Cartan subgroup are displayed. See Section 9.1.

Example 5.1 Let $G=S L(2, \mathbb{C})$, which has a unique inner class of real 
forms. Then $\mathcal{I}_{W}=W \times \Gamma$; we can drop $\Gamma$ and write $\mathcal{I}_{W} / W=\{1, s\}$. There are two conjugacy classes of Cartan subgroups of $S L(2, \mathbb{R})$, compact and split.

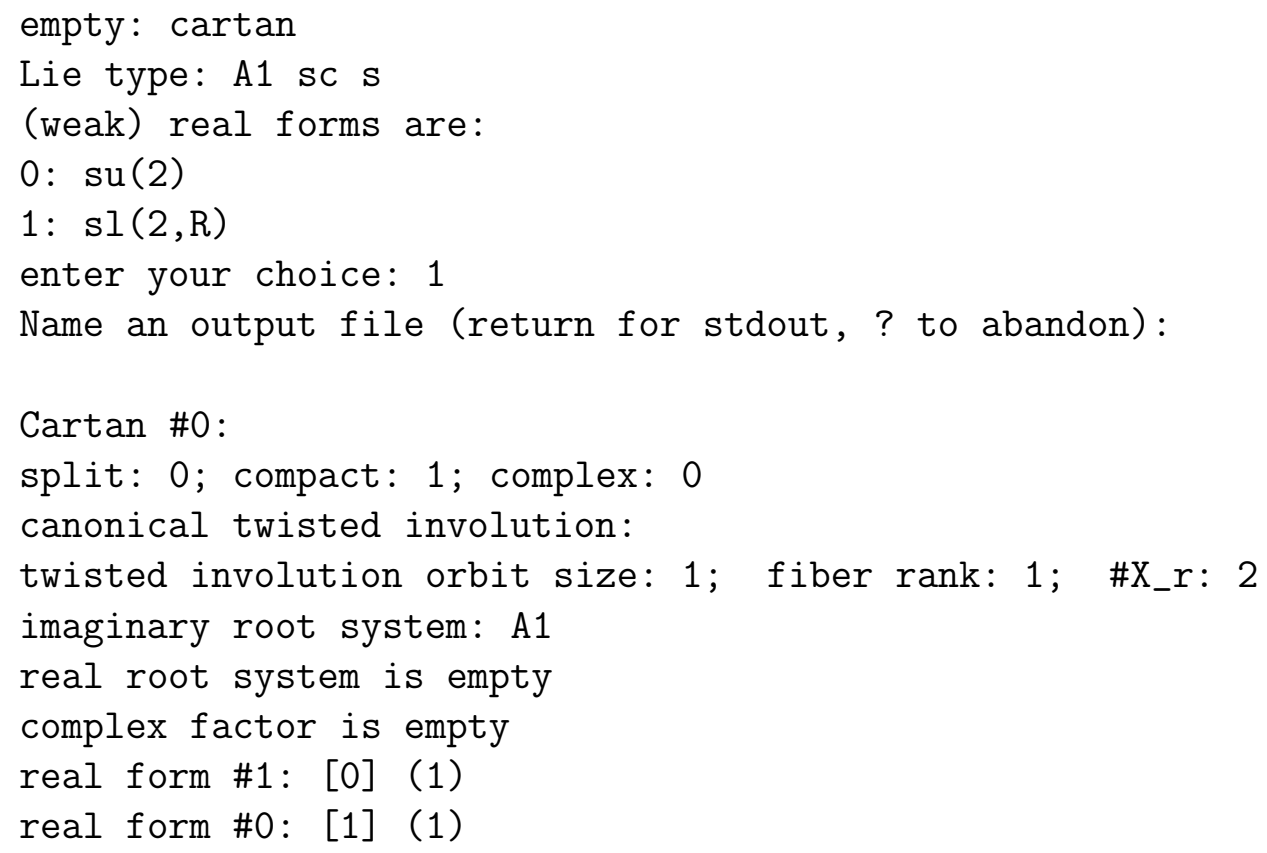

Cartan \#0 is always the fundamental (most compact) Cartan subgroup, in this case $S^{1}$. The corresponding twisted involution is the identity, and its orbit is itself. Both real forms contain this Cartan subgroup.

Cartan \#1 is the split Cartan subgroup $\mathbb{R}^{\times}$, and this occurs only in real form \#1, i.e. $S L(2, \mathbb{R})$.

Example 5.2 Here is the complex group $S L(2, \mathbb{C})$ : 
empty: cartan

Lie type: A1.A1 sc C

there is a unique real form: $\mathrm{sl}(2, \mathrm{C})$

Name an output file (return for stdout, ? to abandon):

Cartan \#0:

split: 0 ; compact: 0 ; complex: 1

canonical twisted involution:

twisted involution orbit size: 2; fiber rank: 0; \#X_r: 2

imaginary root system is empty

real root system is empty

complex factor: A1

real form \#0: [0] (1)

There is one real form, and one conjugacy class of Cartan subgroups, isomorphic to $\mathbb{C}^{\times}$. For a complex group the twisted involutions are in bijection with the Weyl group, so in this case there are 2.

Example 5.3 Here are the Cartan subgroups of $S p(4, \mathbb{R})$. See [1, Example 14.19].

empty: cartan

Lie type: $\mathrm{C} 2 \mathrm{sc} \mathrm{s}$

(weak) real forms are:

$0: \operatorname{sp}(2)$

1: $\operatorname{sp}(1,1)$

2: $\operatorname{sp}(4, R)$

enter your choice: 2

Name an output file (return for stdout, ? to abandon):

Cartan \#0:

split: 0 ; compact: 2 ; complex: 0

canonical twisted involution:

twisted involution orbit size: 1; fiber rank: 2; \#X_r: 4

imaginary root system: B2

real root system is empty

complex factor is empty

real form \#2: $[0,1](2)$ 


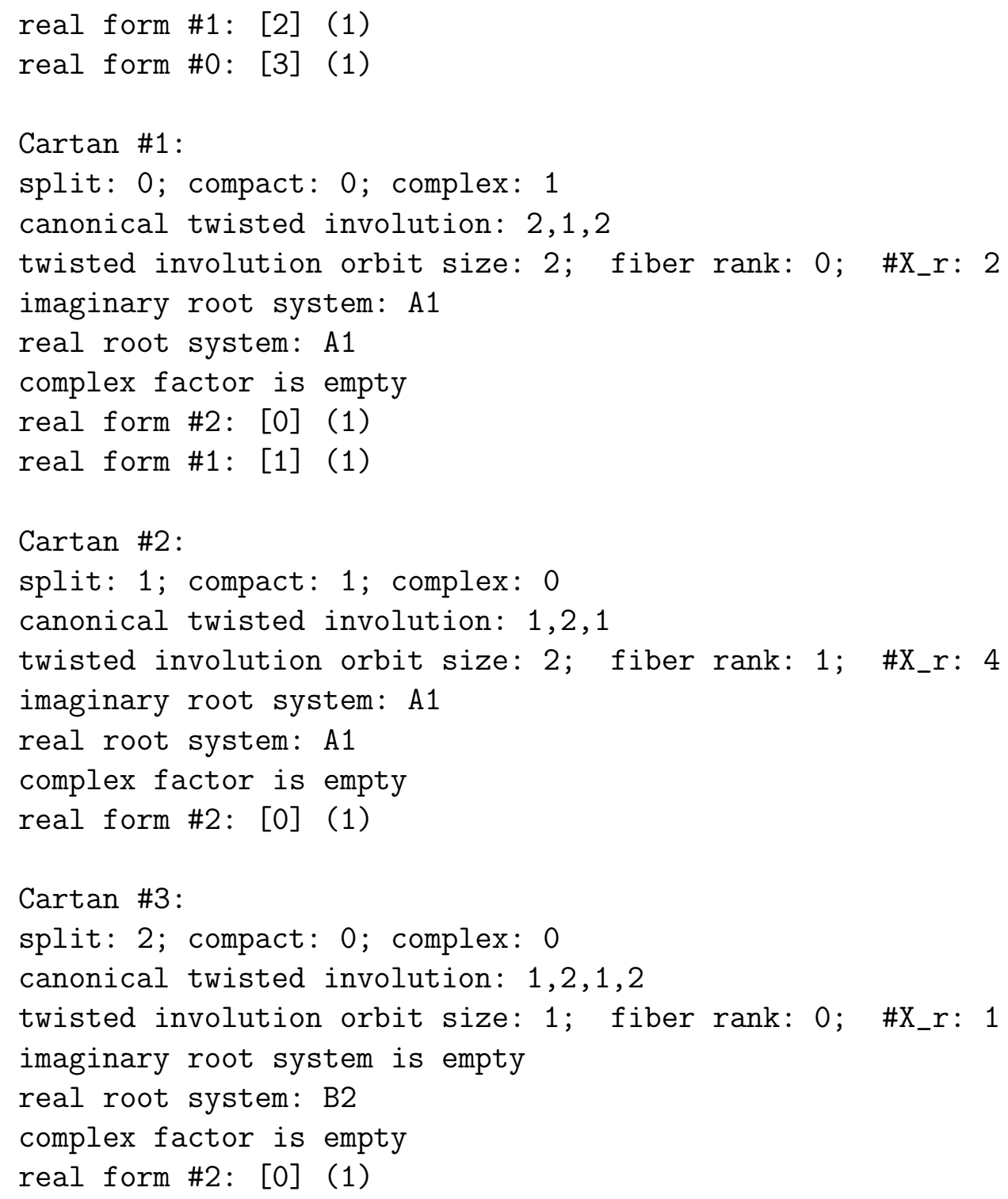

There are four conjugacy classes of Cartan subgroups, isomorphic to $S^{1} \times$ $S^{1}, \mathbb{C}^{\times}, S^{1} \times \mathbb{R}^{\times}$and $\mathbb{R}^{\times} \times \mathbb{R}^{\times}$. All four are contained in the split group $S p(4, \mathbb{R})$; two of them are contained in $S p(1,1)$, and only the compact Cartan subgroup occurs in $S p(2,0)$.

Example 5.4 Here is the real form of $E_{6}$ with $K$ of type $F_{4}$ :

empty: cartan 


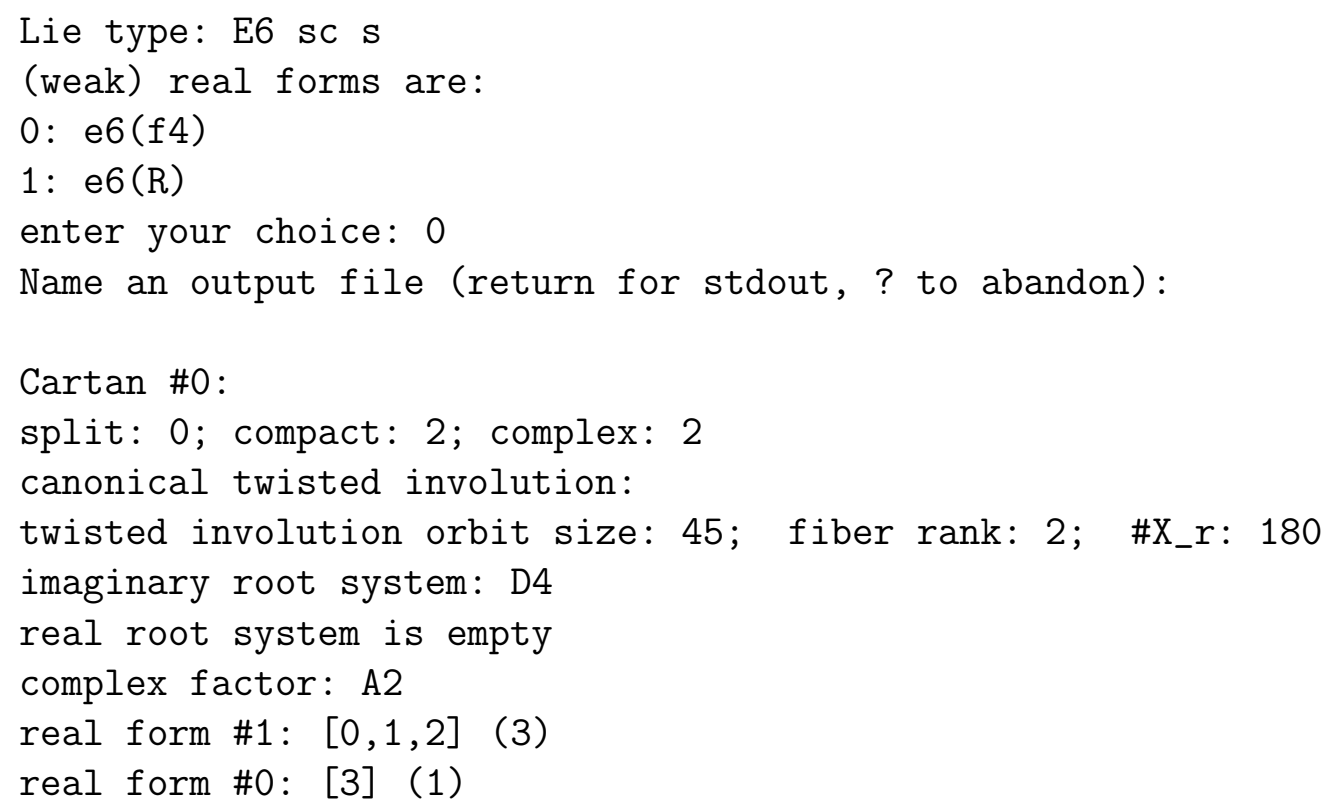

This group has a unique conjugacy class of Cartan subgroups. See Example 8.14 .

We discuss Cartan subgroups of the Classical groups.

Example 5.5 Type $A_{n-1}$. It is most convenient to take $G=G L(n, \mathbb{C})$.

First let $\gamma=1$, so $W^{\Gamma}=S_{n} \times \Gamma$. It is well known that the conjugacy classes of involutions in $S_{n}$ are parametrized by ordered pairs $(a, b) \in \mathbb{N}^{2}$ satisfying $a+2 b=n$ : in cycle notation take $w=(1,2)(3,4) \ldots(2 b-1,2 b)$.

The quasisplit group in this inner class is the unitary group $U(m, m)$ or $U(m+1, m)$. The Cartan subgroup corresponding to $(a, b)$ is isomorphic to $\left(S^{1}\right)^{a} \times\left(\mathbb{C}^{\times}\right)^{b}$; the identity element corresponds to the compact Cartan subgroup.

Now suppose $\gamma$ is given by the unique non-trivial automorphism of the Dynkin diagram $(n \geq 3)$. The split real form is $G L(n, \mathbb{R})$. It turns out that the twisted involutions in $W$ are also parametrized by pairs $(a, b)$ with $a+2 b=n$; in this case the corresponding Cartan subgroup is isomorphic to $\left(\mathbb{R}^{\times}\right)^{a} \times\left(\mathbb{C}^{\times}\right)^{b}$. (This is an aspect of Vogan duality; see Section 8 and $[1$, 1.35 and Corollary 10.9].)

Example 5.6 Types $B_{n}$ and $C_{n}$. 
In this case case $\gamma$ is necessarily trivial, and the Cartan subgroups of $S O(n+1, n)$ or $S p(2 n, \mathbb{R})$ are parametrized by conjugacy classes of involutions in $W \simeq S_{n} \ltimes \mathbb{Z}_{2}^{n}$. These are are parametrized by $(a, b, c)$ with $a+b+2 c=n$, and the corresponding Cartan subgroup is isomorphic to $\left(S^{1}\right)^{a} \times\left(\mathbb{R}^{\times}\right)^{b} \times$ $\left(\mathbb{C}^{\times}\right)^{c}$.

Is is interesting to consider the Hasse diagram of these Cartan subgroups. This is the graph, with one node for each Cartan subgroup, and an edge for each Cayley transform (cf. Section 7) relating two Cartan subgroups. We make a node black if the corresponding Cartan subgroup is the most split Cartan subgroup of a real form of $G$. The split rank is given in the first column. 


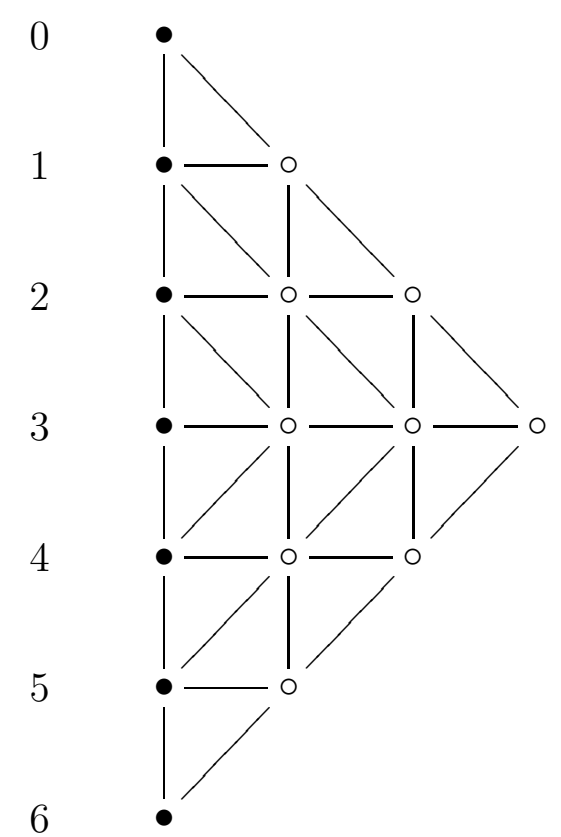

Cartan diagram for $S O(7,6)$

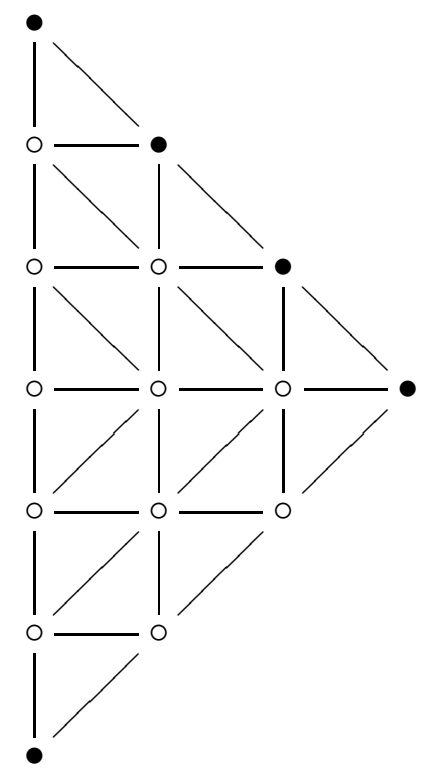

Cartan diagram for $S p(12, \mathbb{R})$

\section{Example 5.7 Type $D_{n}$.}

In this case (for $n \geq 2$ ) there are two choices of $\gamma$, corresponding to the quasisplit groups $S O(n, n)$ and $S O(n+1, n-1)(\gamma=1$ corresponds to $S O(n, n)$ if $n$ is even, and $S O(n+1, n-1)$ if $n$ is odd). It is most convenient to group these two real forms together. Then the Cartan subgroups are parametrized by $(a, b, c)$ with $a+b+2 c=n$, except that $(0,0, c)$ is counted twice. (These two Cartan subgroups are conjugate by the outer automorphism of $S O(n, n)$ coming from $O(n, n)$.) Again the corresponding Cartan subgroup is isomorphic to $\left(S^{1}\right)^{a} \times\left(\mathbb{R}^{\times}\right)^{b} \times\left(\mathbb{C}^{\times}\right)^{c}$. If $a$ is even this Cartan subgroup occurs in $S O(n, n)$ and in $S O(n+1, n-1)$ otherwise. 


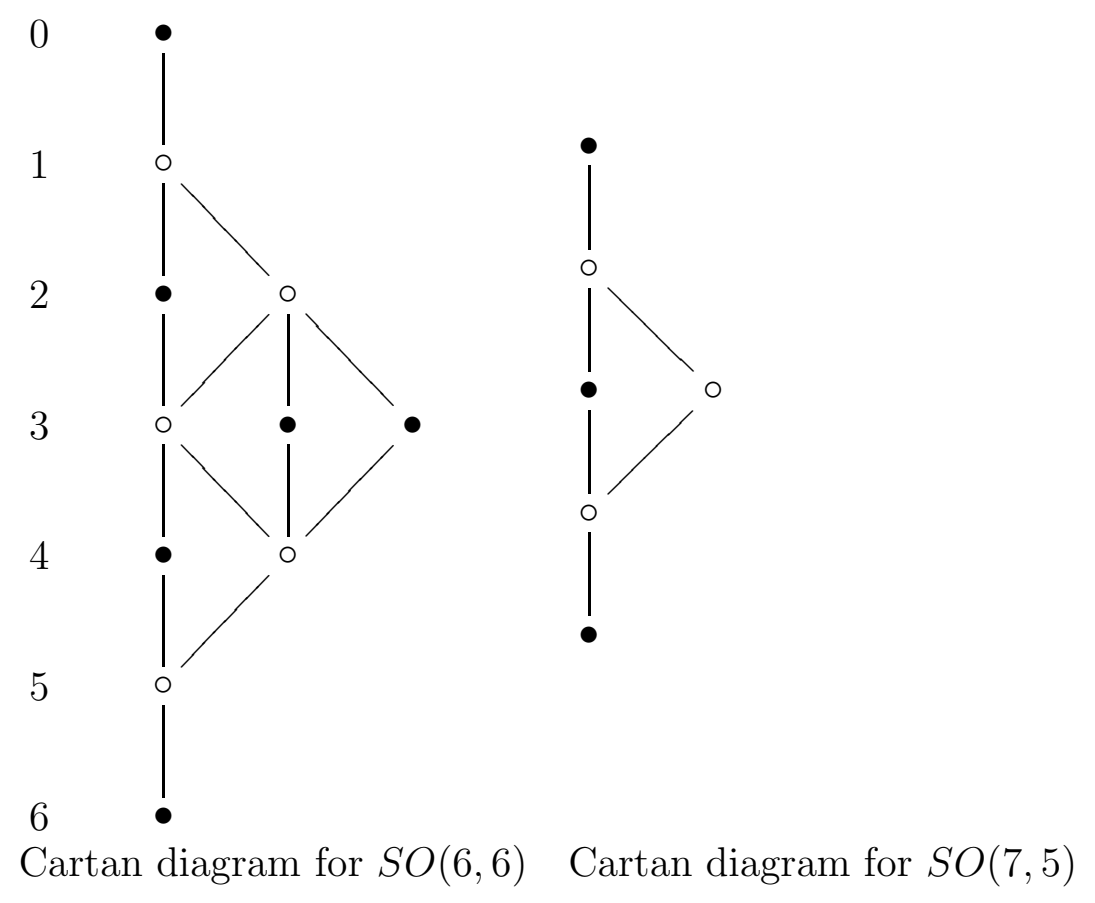

Example 5.8 Note that the Cartan subgroups in $G(\mathbb{R})$ only depend on $W^{\Gamma}$, and are therefore independent of isogeny (this is not true in the p-adic case). While the list of Cartan subgroups is independent of isogeny, the description as a real torus is not, and the nature of the torus can change unexpectedly under isogenies.

For example suppose $G$ is of type $D_{2} \simeq A_{1} \times A_{1}$ and $\gamma \neq 1$. There a unique conjugacy class of Cartan subgroups in this case. In the simply connected case $G(\mathbb{R}) \simeq S L(2, \mathbb{C})$ and the Cartan subgroup is isomorphic to $\mathbb{C}^{\times}$(cf. Example 5.2).

For $S O(3,1)$ we get $S^{1} \times \mathbb{R}^{\times}$:

split: 1 ; compact: 1 ; complex: 0

and for $P S O(3,1) \simeq P S L(2, \mathbb{C})$ we get $\mathbb{C}^{\times}$again:

split: 0 ; compact: 0 ; complex: 1

Note that $G(\mathbb{R})$ is a connected complex group if $G(\mathbb{C})=\operatorname{Spin}(4, \mathbb{C})$ or $P S O(4, \mathbb{C})$, but not $S O(4, \mathbb{C})$. In fact we have: 


$$
\begin{array}{lll}
G & G(\mathbb{R}) & H(\mathbb{R}) \\
\operatorname{Spin}(4, \mathbb{C}) \simeq S L(2, \mathbb{C}) \times S L(2, \mathbb{C}) & \operatorname{Spin}(3,1) \simeq S L(2, \mathbb{C}) & \mathbb{C}^{\times} \\
\operatorname{SO}(4, \mathbb{C}) & \operatorname{SO}(3,1) & \mathbb{R}^{\times} \times S^{1} \\
\operatorname{PSO}(4, \mathbb{C}) \simeq \operatorname{PSL}(2, \mathbb{C}) \times P S L(2, \mathbb{C}) & \operatorname{PSO}(3,1) \simeq \operatorname{PSL}(2, \mathbb{C}) & \mathbb{C}^{\times}
\end{array}
$$

Remark 5.9 If $G(\mathbb{R})$ is a real form of $G L(n, \mathbb{C}), S L(n, \mathbb{C}), S p(2 n, \mathbb{C})$ or $S O(n, \mathbb{C})$ then two Cartan subgroups are isomorphic if and only if they are conjugate by $G(\mathbb{R})$, or by an automorphism of $G(\mathbb{R})$ in the case of $\left(\mathbb{C}^{\times}\right)^{n} \subset$ $S O(2 n, 2 n)$ (cf. Example 5.7). It is perhaps surprising that this fails badly for isogenous groups, as the following example shows.

Example 5.10 Let $G(\mathbb{R})=\operatorname{Spin}(n, n)$ or $P S O(n, n)$ with $n$ even $(n \geq 4)$. There are three non-conjugate Cartan subgroups isomorphic to $\mathbb{R}^{\times} \times S^{1} \times$ $\left(\mathbb{C}^{\times}\right)^{\frac{n}{2}-1}$. Two of these are interchanged by an outer automorphism of $G(\mathbb{R})$. (The corresponding Cartan subgroups of $S O(n, n)$ are isomorphic to $\left(\mathbb{C}^{\times}\right)^{\frac{n}{2}}$.) The third one is not related to the others: it has a different real Weyl group (see the complex factor entry).

Here are the three Cartan subgroups in question for $\operatorname{Spin}(6,6)$.

empty: cartan

Lie type: D6 sc s

(weak) real forms are:

0 : so(12)

1: so $(10,2)$

2: $\operatorname{so} *(12)[1,0]$

3: so* $(12)[0,1]$

4: so $(8,4)$

5: $\operatorname{so}(6,6)$

enter your choice: 5

Name an output file (return for stdout, ? to abandon):

...

Cartan \#4:

split: 1 ; compact: 1 ; complex: 2

canonical twisted involution: $3,4,5,6,4,3,2,3,4,5,6,4,3,1,2,3,4,5,6,4,3,2,1$

twisted involution orbit size: 180; fiber rank: 1; \#X_r: 360

imaginary root system: A1.A1.A1

real root system: $A 1 . A 1 . A 1$

complex factor: A1 
real form \#5: [0] (1)

real form \#4: [1] (1)

Cartan \#5:

split: 1 ; compact: 1 ; complex: 2

canonical twisted involution: $6,4,5,3,4,6,2,3,4,5,1,2,3,4,6$

twisted involution orbit size: 60; fiber rank: 1; \#X_r: 120

imaginary root system: A1.A1.A1

real root system: A1.A1.A1

complex factor: A2

real form \#5: [0] (1)

real form \#3: [1] (1)

Cartan \#6:

split: 1 ; compact: 1 ; complex: 2

canonical twisted involution: $5,4,6,3,4,5,2,3,4,6,1,2,3,4,5$

twisted involution orbit size: 60; fiber rank: 1; \#X_r: 120

imaginary root system: A1.A1.A1

real root system: A1.A1.A1

complex factor: A2

real form \#5: [0] (1)

real form \#2: [1] (1)

...

A similar phenomenon holds in the split real forms of $F_{4}$ and $G_{2}$.

\section{Weyl Groups}

Fix basic data $(G, \gamma), \xi \in \widetilde{\mathcal{X}}$, and set $K=G^{\theta_{\xi}}$ (cf. Section 4). The "real" Weyl group $W(K, H)=\operatorname{Norm}_{K}(H) / H \cap K$ plays an important role. It is isomorphic to $W(G(\mathbb{R}), H(\mathbb{R}))=\operatorname{Norm}_{G(\mathbb{R})}(H(\mathbb{R})) / H(\mathbb{R})$ where $G(\mathbb{R})$ is a real form of $G$ corresponding to $K$, and $H(\mathbb{R})$ is the corresponding real form of $H$. See [1, Section 12].

We briefly recall some constructions from [1, Section 8], also see [4, Proposition 4.16]. Let $\tau$ be the image of $\xi$ in $\mathcal{I}_{W}$. We have

$$
W(K, H) \simeq\left(W_{C}\right)^{\tau} \ltimes\left(W(M \cap K, H) \times W_{r}\right) .
$$

Here 
- $W_{r}$ is the Weyl group of the system of real roots;

- $\left(W_{C}\right)^{\tau}$ is the Weyl group of a certain root system constructed using complex roots ([4, Proposition 3.12]);

- $W(K \cap M, H) \simeq W_{i, c} \ltimes \mathcal{A}(H) \subset W_{i}$, where $W_{i, c}$ is the Weyl group of the root system of compact imaginary roots, and $\mathcal{A}(H)$ is a certain elementary abelian two-group.

See Section 7 for the definition of real and imaginary roots.

To describe $W(K, H)$ it is therefore sufficient to describe $W_{r}, W_{i, c},\left(W_{C}\right)^{\tau}$ and $\mathcal{A}(H)$. The first three are Weyl groups of root systems. The realweyl command describes these three root systems, denoted W_r, W_ic, and $\mathrm{W}^{\wedge} \mathrm{C}$ respectively; and $\mathcal{A}(H)$, denoted A.

Example 6.2 As usual we start with $S L(2, \mathbb{R})$ :

empty: realweyl

Lie type: A1 sc s

(weak) real forms are:

$0: \mathrm{su}(2)$

1: $\operatorname{sl}(2, R)$

enter your choice: 1

cartan class (one of 0,1 ): 0

Name an output file (return for stdout, ? to abandon):

real weyl group is $\mathrm{W}^{\wedge} \mathrm{C} .\left(\left(\mathrm{A} . \mathrm{W}_{-} i \mathrm{C}\right) \times \mathrm{W}^{\wedge} \mathrm{R}\right)$, where:

$\mathrm{W}^{\wedge} \mathrm{C}$ is trivial

$A$ is trivial

W_ic is trivial

$W^{\wedge} R$ is trivial

This is the compact Cartan subgroup, for which $W(K, H)$ is trivial. Here is the split Cartan subgroup:

real: realweyl

cartan class (one of 0,1 ): 1

Name an output file (return for stdout, ? to abandon):

real weyl group is $W^{\wedge} C .\left(\left(A . W_{-} i c\right) x W^{\wedge} R\right)$, where: 


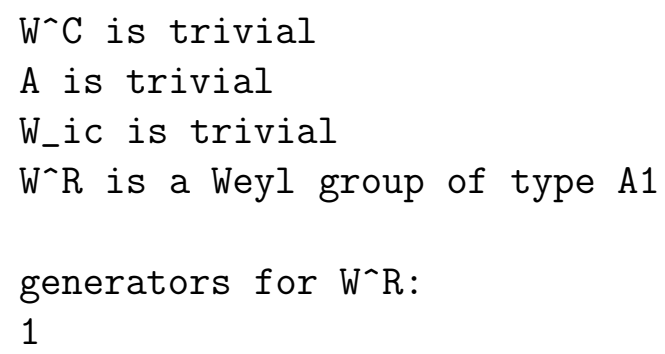

For the split Cartan subgroup the only non-trivial factor is $W_{r}$, and $W(K, H)=$ $W_{r} \simeq \mathbb{Z}_{2}$.

Example 6.3 There is a small change when we compute the Weyl group for the compact Cartan subgroup of $\operatorname{PSL}(2, \mathbb{R})$ instead:

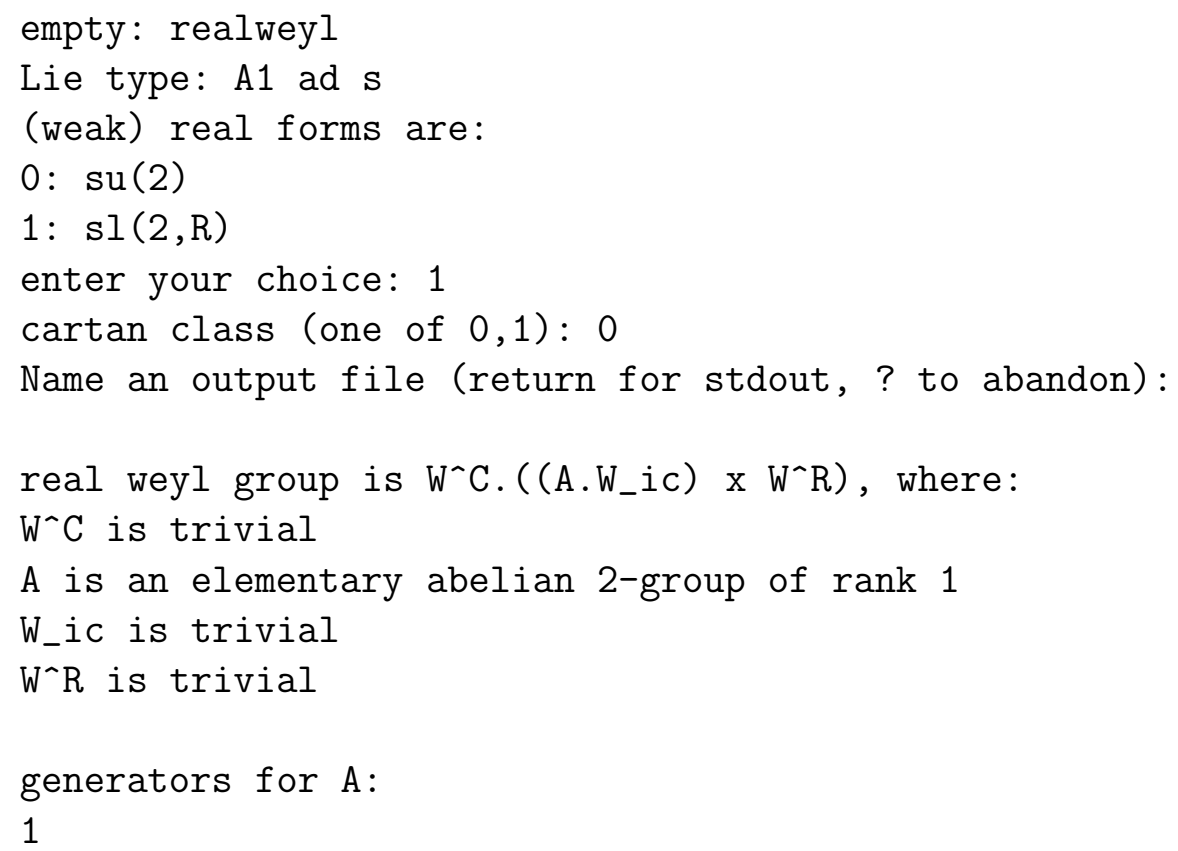

In this case $\mathcal{A}(H)=\mathbb{Z}_{2}$, so $W(K, H)=\mathbb{Z}_{2}$. Recall $P S L(2, \mathbb{R}) \simeq S O(2,1)$ is disconnected; the non-trivial Weyl group element is given by an element in the non-identity component.

Example 6.4 Here is an example, for split groups of type $D_{2 m}$, in which the group $\mathcal{A}(H)$ is quite large. We take the Cartan subgroup $\left(S^{1}\right)^{2} \times\left(\mathbb{C}^{\times}\right)^{m-1}$ 
(cf. Example 5.7). In the notation at the beginning of [1, Section 12] we have

$$
\begin{aligned}
\Delta_{i} & =A_{1}^{m-1} \times D_{2} \simeq A_{1}^{m+1} \\
\Delta_{r} & =A_{1}^{m-1} \\
\Delta_{C} & =A_{m-2} \times A_{m-2} \\
W_{i} & \simeq \mathbb{Z}_{2}^{m+1}, W_{r} \simeq \mathbb{Z}_{2}^{m-1} \\
W_{i, c} & =1 \\
\left(W_{C}\right)^{\tau} & \simeq S_{m-1} \\
W^{\tau} & \simeq S_{m-1} \ltimes\left[\mathbb{Z}_{2}^{m+1} \times \mathbb{Z}_{2}^{m-1}\right] .
\end{aligned}
$$

Here $S_{m-1}$ acts trivially on the final two factors of $\mathbb{Z}_{2}^{m+1}$, and $W^{\tau}$ is the centralizer of $\tau$ in $W$; this group contains $W(K, H)$. So far this discussion is independent of isogeny.

Since $W_{i, c}=1$ the real Weyl group is the same as $W^{\tau}$, with the factor $W_{i}=\mathbb{Z}_{2}^{m+1}$ replaced by $\mathcal{A}(H) \subset W_{i}$. The group $\mathcal{A}(H)$ depends on the isogeny. If $G$ is adjoint it is equal to $W_{i}$. The smallest possible value of $\mathcal{A}(H)$ occurs when $G$ is simply connected. It isn't as easy to compute $\mathcal{A}(H)$ in this case; the software will tell us.

For example consider the split group $\operatorname{Spin}(8,8)$ of type $D_{8}(m=4)$. The Cartan class is \#5:

\section{Cartan \#5:}

split: 0 ; compact: 2 ; complex: 3 canonical twisted involution: $6,7,8,6,5$, [truncated] twisted involution orbit size: 3360; fiber rank: 2; \#X_r: 13440 imaginary root system: A1.A1.A1.A1.A1 real root system: A1.A1.A1

complex factor: A2

..

and here is the relevant output from realweyl:

real weyl group is $W^{\wedge} C .\left(\left(A . W_{-} i c\right) \times W^{\wedge} R\right)$, where:

$W^{\wedge} C$ is isomorphic to a Weyl group of type $A 2$

$A$ is an elementary abelian 2-group of rank 3

W_ic is trivial

$W^{\wedge} R$ is a Weyl group of type A1.A1.A1 
Thus $\mathcal{A}(H) \simeq \mathbb{Z}_{2}^{m-1}$.

If $H$ is a fundamental (most compact) Cartan subgroup then $H \cap K$ is a Cartan subgroup of $K$, and it is not hard to see that $W(G, H) \simeq W(K, H \cap$ $K)$. In particular if $K$ is connected this is the Weyl group of the root system of $H \cap K$ in $K$. It is interesting to consider this in the case of real forms of $E_{6}$ of unequal rank.

Example 6.6 First consider $E_{6}\left(F_{4}\right)$ :

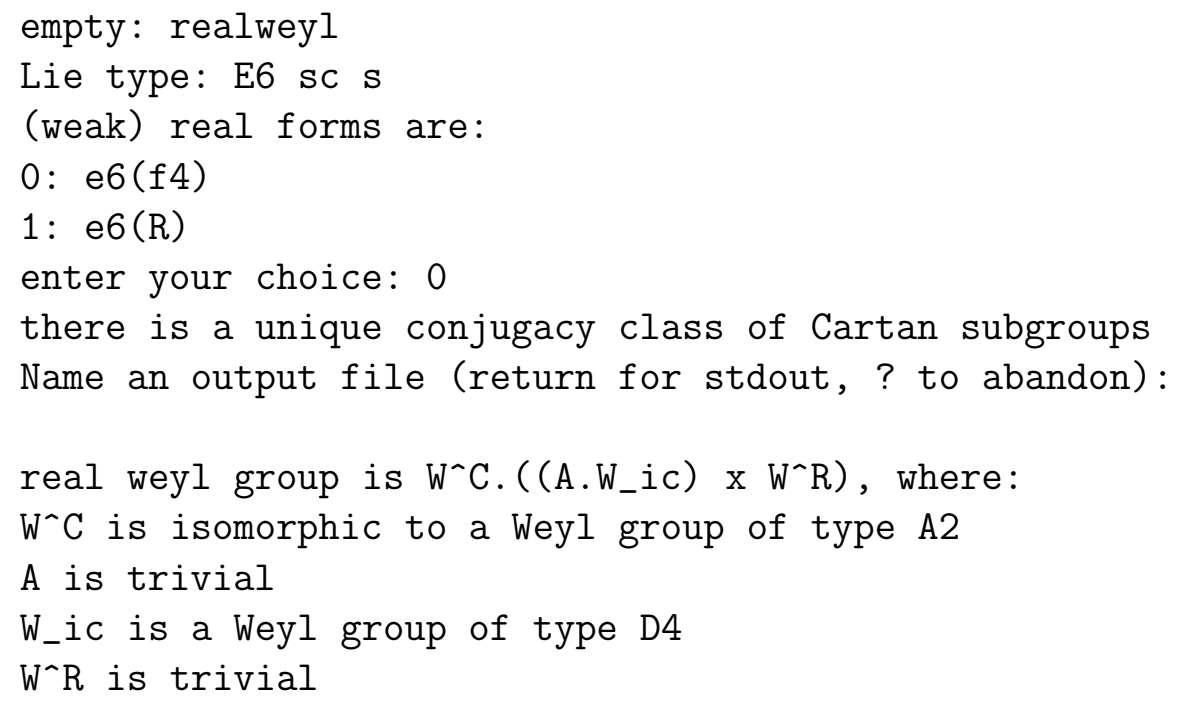

Thus $W(K, H) \simeq W\left(A_{2}\right) \ltimes W\left(D_{4}\right) \simeq S_{3} \ltimes W\left(D_{4}\right)$. The action of $S_{3}$ on $W\left(D_{4}\right)$ is induced by the action of $S_{3}$ on the Dynkin diagram of $D_{4}$. By the remarks above we have obtained the classical isomorphism

$$
W\left(F_{4}\right) \simeq S_{3} \ltimes W\left(D_{4}\right) .
$$

Example 6.8 Something similar happens for the split real form of $E_{6}$, in which $K$ is of type $C_{4}$. In this case realweyl gives:

real weyl group is $W^{\wedge} C .\left(\left(A . W_{-} i c\right) \times W^{\wedge} R\right)$, where:

$\mathrm{W}^{\wedge} \mathrm{C}$ is isomorphic to a Weyl group of type A2

$A$ is an elementary abelian 2-group of rank 2

W_ic is a Weyl group of type A1.A1.A1.A1

$W^{\wedge} R$ is trivial 
Thus

$$
\begin{aligned}
W\left(C_{4}\right) & \simeq S_{3} \ltimes\left(\mathbb{Z}_{2}^{2} \times \mathbb{Z}_{2}^{4}\right) \\
& \simeq S_{4} \ltimes \mathbb{Z}_{2}^{4} .
\end{aligned}
$$

Example 6.10 Complex groups.

Fix basic data $\left(G=G_{1} \times G_{1}, \gamma\right)$ for some group $G_{1}$, with $\gamma$ the inner class of the complex group ( $\gamma$ switches the two factors). Thus $G(\mathbb{R})=G_{1}(\mathbb{C})$ and $K=G_{1}^{\Delta}$ (the diagonal embedding). If $H_{1}$ is a Cartan subgroup of $G_{1}$ then $H=H_{1} \times H_{1}$ is a Cartan subgroup of $G, W(G, H) \simeq W\left(G_{1}, H_{1}\right) \times W\left(G_{1}, H_{1}\right)$, and $W(K, H)=W\left(G_{1}, H_{1}\right)^{\Delta}$.

In the notation of the output of realweyl all terms are trivial except $\mathrm{W}^{\wedge} \mathrm{C}$, which equals $W\left(G_{1}, H_{1}\right)$.

empty: type

Lie type: A3.A3 sc C

main: realweyl

there is a unique real form: $\mathrm{sl}(4, \mathrm{C})$

there is a unique conjugacy class of Cartan subgroups

Name an output file (hit return for stdout):

real weyl group is $W^{\wedge} C .\left(\left(A . W_{-} i c\right) \times W^{\wedge} R\right)$, where:

$\mathrm{W}^{\wedge} \mathrm{C}$ is isomorphic to a Weyl group of type $\mathrm{A} 3$

$A$ is trivial

W_ic is trivial

$W^{\wedge} R$ is trivial

generators for $\mathrm{W}^{\wedge} \mathrm{C}$ :

2,5

3,6

1,4

Simple roots 1,2,3 give the first copy of $G_{1}$, and 4,5,6 give the second. The elements $s_{1} s_{4}, s_{2} s_{5}$ and $s_{3} s_{6}$ generate $W\left(G_{1}, H_{1}\right)^{\Delta}=W\left(A_{3}\right)=S_{4}$. 


\section{$7 \quad K$ orbits on the flag variety and $\mathcal{X}^{r}$}

Fix basic data $(G, \gamma)$ (Section 2) and let $\mathcal{X}^{r}$ be the reduced parameter space (Section 4). Given $x \in \mathcal{X}^{r}$ let

$$
\mathcal{X}^{r}[x]=\left\{x^{\prime} \in \mathcal{X}^{r} \mid x^{\prime} \text { is } G \text {-conjugate to } x\right\}
$$

(see $[1,9.7])$. Choose a preimage $\xi$ of $x$ in $\tilde{\mathcal{X}}^{r}$ and let $\theta=\operatorname{int}(\xi), K=G^{\theta}$. Then $\mathcal{X}^{r}[x]$ is isomorphic to $K \backslash G / B$. In fact, one of the important properties of $\mathcal{X}^{r}$ is that it captures information about the $K$ orbits on $G / B$ for all $K$ in this inner class. Recall (4.4) $\left\{\xi_{i} \mid 1 \leq i \leq n\right\}$ is a set of representatives of the strong real forms in this inner class, and for each $i \in I$ we let $K_{i}=K_{\xi_{i}}$. Then [1, Corollary 9.9]:

$$
\mathcal{X}^{r}=\coprod_{i=1}^{n} \mathcal{X}\left[x_{i}\right] \simeq \coprod_{i=1}^{n} K_{i} \backslash G / B .
$$

The structure of $K_{i} \backslash G / B$ is determined to a large extent by the cross action (cf. Section 4), and Cayley transforms, which appear in the space $\mathcal{X}^{r}$ as follows. Fix $x \in \mathcal{X}^{r}$ and let $\tau=p(x) \in \mathcal{I}_{W}$. Then $\tau$ acts on the roots, and a root $\alpha$ is classified as imaginary, real or complex with respect to $\tau$ if $\tau(\alpha)=\alpha,-\alpha$ or neither, respectively. Let $\xi$ be a preimage of $x$ in $\tilde{\mathcal{X}}^{r}$. If $\alpha$ is imaginary we say it is compact or noncompact with respect to $x$ if this holds with respect to $\theta_{\xi}$ (independent of the choice of $\xi$ ). See [1, Section 14] for details.

Now suppose $\alpha$ is a noncompact imaginary root with respect to $x$. Then associated to $\alpha$ and $x$ is a new element of $\mathcal{X}^{r}$ denoted $c^{\alpha}(x)$. It satisfies $p\left(c^{\alpha}(x)\right)=s_{\alpha} p(x)$, and $\alpha$ is real with respect to $s_{\alpha} \tau$. Its inverse is the single or double-valued real Cayley transform $c_{\alpha}(x)$ : if $\alpha$ is real with respect to $x$ then $c_{\alpha}(x)$ is a set with one or two elements.

The (finite) set of orbits of $K$ on $G / B$ is described by the kgb command.

Example 7.1 We first consider $S L(2, \mathbb{R})$ and $P G L(2, \mathbb{R})$. See $[1$, Example $12.20]$.

main: kgb

(weak) real forms are:

$0: \mathrm{su}(2)$

1: $\operatorname{sl}(2, R)$ 


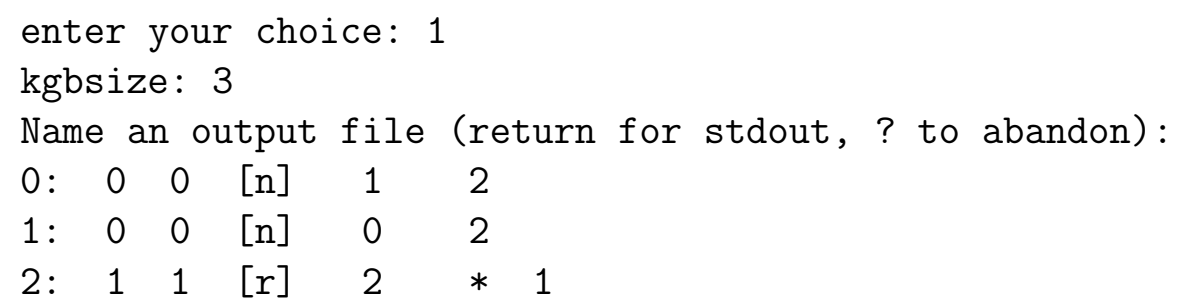

Here $G(\mathbb{R})=S L(2, \mathbb{R}), K(\mathbb{R})=S^{1}$ and $K=K(\mathbb{C})=\mathbb{C}^{\times}$. This acts on $G / B=P^{1}(\mathbb{C})=\mathbb{C} \cup \infty$ by $\mathbb{C}^{\times} \ni z: w \rightarrow z^{2} w$. The entry kgbsize gives the number of orbits, 3 in this case. These are $0, \infty$ and $\mathbb{C}^{\times}$, labelled \#0,1,2 respectively (the first entry in each line) in the output of kgb. (We describe other parts of the output below).

Here is the result for $P G L(2, \mathbb{R})$; see [1, Example 12.25] for more detail.

$\begin{array}{lllllll}0: & 0 & 0 & {[\mathrm{n}]} & 0 & 1 & \\ 1: & 1 & 1 & {[\mathrm{r}]} & 1 & * & 1\end{array}$

In this case $G / B$ is the same, but $K=O(2, \mathbb{C})$; an element from the nonidentity component identifies 0 and $\infty$, so there are only two orbits.

We now explain the other information given in the output. Fix a row $i$ with corresponding $x_{i} \in \mathcal{X}^{r}[x] \simeq K_{\xi} \backslash G / B$, and write $\mathcal{O}_{i}$ for the corresponding orbit of $K_{\xi}$ on $G / B$.

The image of $x_{i}$ in $\mathcal{I}_{W}$ corresponds to a Cartan subgroup; the first entry gives the number of this Cartan subgroup in the output of the cartan command. The second number is the length of this orbit, which is $\operatorname{dim}\left(\mathcal{O}_{i}\right)-$ $\operatorname{dim}\left(\mathcal{O}_{0}\right)$. In the equal rank case $(\gamma=1) \operatorname{dim}\left(\mathcal{O}_{0}\right)=0$.

The simple roots are labelled $1, \ldots, n$. The term in brackets in row $\mathrm{i}$ of the output gives the type of each simple root: $r, \mathrm{C}, \mathrm{n}, \mathrm{c}$ for real, complex, noncompact imaginary, or compact imaginary, respectively.

The next $n$ columns give the cross action of the simple roots. An entry $j$ in column $\mathrm{k}$ of row $\mathrm{i}$ means that the the cross action of the $k^{\text {th }}$ simple root takes $x_{i}$ to $x_{j}$.

The next $n$ columns give Cayley transforms by noncompact imaginary roots. There is an entry in in column $\mathrm{k}$ of row $\mathrm{i}$ only if the $k^{\text {th }}$ simple root is noncompact imaginary for $x_{i}$; in this case an entry $\mathrm{j}$ means this Cayley transform takes $x_{i}$ to $x_{j}$. These Cayley transforms are single valued (the inverse, multivalued Cayley transforms are not listed). 
The final entry is the twisted involution in $W$ corresponding to $x_{i}$, as a product of simple reflections (cf. Section 4).

Here is the $S L(2, \mathbb{R})$ example again:

$\begin{array}{lllllll}0: & 0 & 0 & {[\mathrm{n}]} & 1 & 2 & \\ 1: & 0 & 0 & {[\mathrm{n}]} & 0 & 2 & \\ 2: & 1 & 1 & {[\mathrm{r}]} & 2 & * & 1\end{array}$

Here is the information we can read off from this output. Orbits \#0 and \#1 correspond to the compact Cartan subgroup (\#0), and are 0-dimensional. Orbit \#2 corresponds to the split Cartan subgroup and is one dimensional. The first two orbits are interchanged by the cross action of the simple root. For orbits \#0,\#1 this root is noncompact, and the Cayley transform takes each of these orbits to orbit \#2.

See [1, Example 14.19] for a detailed discussion of $\operatorname{Sp}(4, \mathbb{R})$.

Example 7.2 Here is $S L(3, \mathbb{R})$ :

empty: kgb

Lie type: A2 sc s

there is a unique real form: $s l(3, R)$

kgbsize: 4

Name an output file (return for stdout, ? to abandon):

$0: \quad 0 \quad 00 \quad[\mathrm{C}, \mathrm{C}] \quad 2 \quad 1 \quad * \quad *$

1: $10 \quad[n, C] \quad 1 \quad 0 \quad 3 * 2,1$

2: $100 \quad[\mathrm{C}, \mathrm{n}] \quad 0 \quad 2 \quad * \quad 3 \quad 1,2$

3: $21 \quad[r, r] \quad 3 \quad 3 * * \quad * 1,2,1$

This is not an equal rank case; the dimension of the closed orbit is 1 , and the dimension of the unique open orbit is $1+2=3$, the dimension of $G / B$.

Example 7.3 We illustrate one way to compute the order of $\mathcal{X}^{r}$ using this information. Consider equal rank real forms of $S L(4, \mathbb{C})$, i.e. $S U(4), S U(3,1)$ and $S U(2,2)$.

Using kgb we compute the number of orbits in each case, given by kgbsize. The result is:

\begin{tabular}{|c|c|}
\hline$G$ & $|K \backslash G / B|$ \\
\hline$S U(2,2)$ & 21 \\
\hline$S U(3,1)$ & 10 \\
\hline$S U(4,0)$ & 1 \\
\hline
\end{tabular}


We will see later (Example 9.3) there are two strong real forms mapping to $S U(4)$ and $S U(3,1)$, and one mapping to $S U(2,2)$. This means that the set $K \backslash G / B$ for $S U(3,1)$, of order 10, appears twice in $\mathcal{X}^{r}$, and similary for $S U(4)$. Therefore the order of $\mathcal{X}^{r}$ is

$$
2 \times 1+2 \times 10+1 \times 21=43 .
$$

This agrees with the counting done a different way in Example 9.3.

Example 7.5 We do the same example again for equal rank forms of the adjoint group $P S L(4, \mathbb{C})$.

\begin{tabular}{|c|c|}
\hline$G$ & $|K \backslash G / B|$ \\
\hline$P U(2,2)$ & 12 \\
\hline$P U(3,1)$ & 10 \\
\hline$P U(4,0)$ & 1 \\
\hline
\end{tabular}

Since $G$ is adjoint the map from strong real forms to real forms is bijective, so $\mathcal{X}^{r}$ has order $12+10+1=23$. See Example 9.1

Example 7.6 If $G=S p(4, \mathbb{R})$ there are 11 orbits of $K$ on $G / B, 4$ of which are closed.

\begin{tabular}{|c|c|c|c|c|c|c|c|}
\hline $0: 0$ & 0 & {$[\mathrm{n}, \mathrm{n}]$} & 1 & 2 & 6 & 4 & \\
\hline 1: & 0 & {$[\mathrm{n}, \mathrm{n}]$} & 0 & 3 & 6 & 5 & \\
\hline 2: & 0 & {$[c, n]$} & 2 & 0 & $*$ & 4 & \\
\hline 3: & 0 & {$[c, n]$} & 3 & 1 & $*$ & 5 & \\
\hline 4: & 2 & {$[C, r]$} & 8 & 4 & $*$ & $*$ & 2 \\
\hline 5: & 2 & {$[C, r]$} & 9 & 5 & $*$ & $*$ & 2 \\
\hline 6: & 1 & {$[r, C]$} & 6 & 7 & $*$ & $*$ & 1 \\
\hline 7: & 1 & {$[\mathrm{n}, \mathrm{C}]$} & 7 & 6 & 10 & $*$ & $2,1,2$ \\
\hline 8: & 2 & {$[C, n]$} & 4 & 9 & $*$ & 10 & $1,2,1$ \\
\hline 9: & 2 & {$[\mathrm{C}, \mathrm{n}]$} & 5 & 8 & $*$ & 10 & $1,2,1$ \\
\hline : 3 & 3 & {$[r, r]$} & 10 & 10 & $*$ & $*$ & $1,2,1,2$ \\
\hline
\end{tabular}

For $G=P S p(4, \mathbb{R})=S O(3,2)$ there are 7 orbits, 2 of them closed:

$\begin{array}{lllllllll}0: & 0 & 0 & {[\mathrm{n}, \mathrm{n}]} & 0 & 1 & 3 & 2 & \\ 1: & 0 & 0 & {[\mathrm{c}, \mathrm{n}]} & 1 & 0 & * & 2 & \\ 2: & 1 & 2 & {[\mathrm{C}, \mathrm{r}]} & 5 & 2 & * & * & 2 \\ 3: & 1 & 1 & {[\mathrm{r}, \mathrm{C}]} & 3 & 4 & * & * & 1\end{array}$




$\begin{array}{lllllllll}4: & 2 & 1 & {[\mathrm{n}, \mathrm{C}]} & 4 & 3 & 6 & * & 2,1,2 \\ 5: & 2 & 2 & {[\mathrm{C}, \mathrm{n}]} & 2 & 5 & * & 6 & 1,2,1 \\ 6: & 3 & 3 & {[\mathrm{r}, \mathrm{r}]} & 6 & 6 & * & * & 1,2,1,2\end{array}$

See Example 8.13.

Example 7.7 Complex Groups.

As in Example 6.10 let $G=G_{1} \times G_{1}, K=G_{1}^{\Delta}$, and $G(\mathbb{R})=G_{1}(\mathbb{C})$. Then $B=B_{1} \times B_{1}$. It is well known, and easy to see, that $(g, h) \rightarrow g h^{-1}$ induces a bijection

$$
G_{1}^{\Delta} \backslash G_{1} \times G_{1} / B_{1} \times B_{1} \simeq B_{1} \backslash G_{1} / B_{1}
$$

By the Bruhat decomposition the right hand side is parametrized by the Weyl group of $G_{1}$.

For example take $G(\mathbb{R})=G_{1}(\mathbb{C})=S L(3, \mathbb{C})$.

main: type

Lie type: A2.A2 sc C

main: $\mathrm{kgb}$

there is a unique real form: $\mathrm{sl}(3, \mathrm{C})$

kgbsize: 6

Name an output file (hit return for stdout):

$0: \quad 2 \quad 1 \quad 2 \quad 1 \quad * \quad * \quad * \quad * \quad$ [CCCC $] \quad 0$

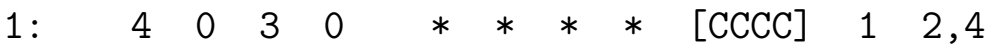

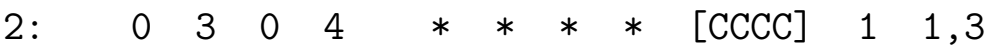

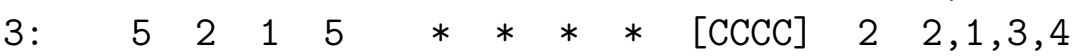

4: $1 \quad 1 \quad 5 \quad 5 \quad 2 \quad * \quad * \quad * \quad * \quad$ [CCCC] 2 1,2, 4, 3

5: $\quad 3 \quad 4 \quad 4 \quad 3 \quad * \quad * \quad * \quad * \quad$ [CCCC] $31,2,1,3,4,3$

Since all roots are complex there are no Cayley transforms. The cross action of the Weyl group $W\left(A_{2}\right) \simeq S^{3}$ is simply transitive. The last column gives the Weyl group element $(w, w)$ as a product of simple reflections; just taking the first half of each entry we see $W=\left\{i d, s_{2}, s_{1}, s_{2} s_{1}, s_{1} s_{2}, s_{1} s_{2} s_{1}\right\}$. See Example 6.10. 


\section{Representation Theory: Blocks and the two- sided parameter space}

The final ingredient in the algorithm is the two-sided parameter space. Let $G^{\vee}$ be the dual group of $G$. The involution $\gamma \in \operatorname{Out}(G)$ defines an involution $\gamma^{\vee} \in \operatorname{Out}\left(G^{\vee}\right)$ and $\left(G^{\vee}, \gamma^{\vee}\right)$ is also basic data. See [1, Section 2] for details. Let $\mathcal{X}^{\vee}$ be the one-sided parameter space defined by $\left(G^{\vee}, \gamma^{\vee}\right)$.

Suppose $x \in \mathcal{X}$ and $\xi \in \widetilde{\mathcal{X}}$ is a pre-image of $x$ in $\widetilde{\mathcal{X}}$. Then $\theta_{\xi}$ restricted to $H$ is independent of the choice of $\xi$, and we denote it $\theta_{x, H}$. We also write $\theta_{x, H} \in \operatorname{End}(\mathfrak{h})$ for its differential. There is a natural pairing between $\mathfrak{h}$ and $\mathfrak{h}^{\vee}$ (a Cartan subalgebra on the dual side); the adjoint $\theta_{x, H}^{t}$ of $\theta_{x, H}$ is an element of $\operatorname{End}\left(\mathfrak{h}^{\vee}\right)$.

We can now define the two-sided parameter space:

$$
\mathcal{Z}=\left\{(x, y) \in \mathcal{X} \times \mathcal{X}^{\vee} \mid \theta_{x, H}^{t}=-\theta_{y, H^{\vee}}\right\} .
$$

See [1, Section 10]. We define the reduced two-sided parameter space $\mathcal{Z}^{r}$ by replacing $\mathcal{X}$ and $\mathcal{X}^{\vee}$ with the corresponding reduced spaces (cf. Section 4). This is a finite set.

With the obvious notation $W \times W^{\vee}$ acts on $\mathcal{Z}$ and $\mathcal{Z}^{r}$; this is the cross action. By the condition relating $x$ and $y$ in (8.1), if $\alpha$ is a real root with respect to $x$ then $\alpha^{\vee}$ is an imaginary root with respect to $y$, and vice-versa. Thus Cayley transforms are defined on $\mathcal{Z}^{r}$, via a real root on one side, and an imaginary noncompact root on the other.

The main result in [1], Theorem 10.3, (also see [1, Theorem 7.15]), is that the space $\mathcal{Z}$ parametrizes the irreducible representations of strong real forms of $G$ "up to translation"; equivalently with certain regular integral infinitesimal characters. Because of our emphasis on the reduced parameter space, we give a slightly different version here.

Apply the construction in the paragraph preceding (4.1) to choose a subset $Z^{\vee r}$ of the center $Z^{\vee}$ of $G^{\vee}$. The pairing of $\mathfrak{h}$ and $\mathfrak{h}^{\vee}$ gives an isomorphism $\mathfrak{h}^{*} \simeq \mathfrak{h}^{\vee}$, and let

$$
L=\left\{\lambda \in \mathfrak{h}^{*} \mid \exp (2 \pi i \lambda) \in Z^{\vee r}\right\} .
$$

Note that $L$ is a subset of the weight lattice $P=\left\{\lambda \in \mathfrak{h}^{*} \mid\left\langle\lambda, \alpha^{\vee}\right\rangle \in \mathbb{Z}\right\}$. Choose a set $\Lambda$ of representatives of $L / X^{*}(H)$, satisfying $\left\langle\lambda, \alpha^{\vee}\right\rangle \neq 0$ for all $\lambda \in \Lambda$ and all roots $\alpha$. This is a finite set. 
Choose a set $\left\{\xi_{i} \mid 1 \leq i \leq n\right\}$ as in (4.4) For each $i$ let $G_{i}(\mathbb{R})$ be the real form of $G$ defined by $\xi_{i}$, and let $\Pi\left(G_{i}(\mathbb{R}), \Lambda\right)$ be the set of irreducible admissible representations of $G_{i}(\mathbb{R})$ with infinitesimal character contained in $\Lambda$.

Theorem 8.3 There is a natural bijection

$$
\mathcal{Z}^{r} \stackrel{1-1}{\longleftrightarrow} \coprod_{i=1}^{n} \Pi\left(G_{i}(\mathbb{R}), \Lambda\right)
$$

This differs from [1, Theorem 10.3] in several ways. First of all on the left hand side we're using $\mathcal{Z}^{r}$ instead of $\mathcal{Z}$. On the right hand side the union is over strong real forms in the sense of the reduced parameter space, instead of the possibly infinite set $\mathcal{I} / G$ of $[1,5.15]$. Finally the set $\Lambda=L / X^{*}(H)$ is contained in the corresponding set $P / X^{*}(H)$ of [1].

Fix $(x, y) \in \mathcal{Z}^{r}$, and consider the representations associated to the pairs $\left(x^{\prime}, y^{\prime}\right)$ in the subset $\mathcal{X}^{r}[x] \times \mathcal{X}^{\vee, r}[y]$ of $\mathcal{Z}^{r}$. Choose $i$ so that $x$ is $G$-conjugate to $x_{i}$. Then these are representations of $G_{i}(\mathbb{R})$, all with the same infinitesimal character. In fact this set of irreducible representations is a block in the sense of [3, Chapter 9], and every block is obtained this way.

The entire construction is obviously symmetric in $G$ and $G^{\vee}$, so an element $(x, y) \in \mathcal{Z}^{r}$, which defines a representation $\pi$ of a real form of $G$, also defines a representation $\pi^{\vee}$ of a real form of $G^{\vee}$. The map $\pi \rightarrow \pi^{\vee}$ is a version of Vogan Duality; see [4] and [1, 1.35 and Corollary 10.9]. In particular this is a duality of blocks: the set $\mathcal{X}^{r}[x] \times \mathcal{X}^{\vee r}[y]$ defines a block $\mathcal{B}$ of a real form of $G$, a block $\mathcal{B}^{\vee}$ of a real form of $G^{\vee}$, and gives a bijection $\mathcal{B} \leftrightarrow \mathcal{B}^{\vee}$.

Before we look at individual representations, it is helpful to look at the sizes of blocks, which are given by the blocksizes command.

Example 8.5 Here are the blocks for real forms of $S L(2, \mathbb{C})$ :

main: type

Lie type: A1 sc s

main: blocksizes

$\begin{array}{ll}0 & 1\end{array}$

13 
The rows and columns correspond to real forms of $G$ and $G^{\vee}$, respectively. Adding some labelling by hand the picture is:

$\begin{array}{lll} & \mathrm{SO}(3) & \mathrm{SO}(2,1) \\ \mathrm{SU}(2) & 0 & 1 \\ \mathrm{SL}(2, \mathrm{R}) & 1 & 3\end{array}$

Thus $S U(2)$ has a single block, dual to a block of $S O(2,1)$, and $S L(2, \mathbb{R})$ has two blocks, dual to $S O(3)$, and $S O(2,1)$, respectively. See Example 8.11.

Example 8.6 The corresponding output for $P S L(2, \mathbb{C})$ is the same, but there is a subtle point here.

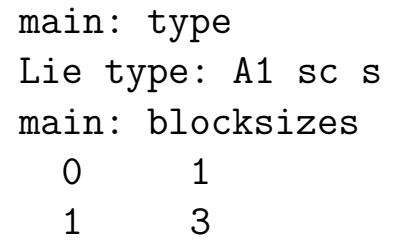

Since the dual group $S L(2, \mathbb{C})$ is not adjoint, the map from strong real forms to real forms (on the dual side) is not injective. There are two strong real forms mapping to $S U(2)$, and we can label the strong real forms $S U(2,0), S U(1,1)$ and $S U(0,2)$. Consequently there are two blocks of $S O(2,1)$ of size 1. See Example 8.12. We could display this information by constructing a table by hand showing strong real forms on the dual side:

$\begin{array}{llll} & \mathrm{SU}(2,0) & \mathrm{SU}(0,2) & \mathrm{SL}(2, \mathrm{R}) \\ \mathrm{SO}(3) & 0 & 0 & 1 \\ \mathrm{SO}(2,1) & 1 & 1 & 3\end{array}$

Blocks tend to be concentrated on the quasisplit forms of $G$ or $G^{\vee}$.

Example 8.7 Here is the output of blocksizes for real forms of $S p(12, \mathbb{C})$, with the real forms added:

$\begin{array}{llllllll} & \mathrm{SO}(13) & \mathrm{SO}(12,1) & \mathrm{SO}(11,2) & \mathrm{SO}(10,3) & \mathrm{SO}(9,4) & \mathrm{SO}(8,5) & \mathrm{SO}(7,6) \\ \mathrm{Sp}(6) & 0 & 0 & 0 & 0 & 0 & 0 & 1 \\ \mathrm{Sp}(5,1) & 0 & 0 & 0 & 0 & 0 & 0 & 36 \\ \mathrm{Sp}(4,2) & 0 & 0 & 0 & 0 & 0 & 0 & 315 \\ \mathrm{Sp}(3,3) & 0 & 0 & 0 & 0 & 0 & 0 & 680 \\ \mathrm{Sp}(12, \mathrm{R}) & 1 & 13 & 108 & 556 & 1975 & 4707 & 7416\end{array}$


Example 8.8 In the case of equal rank real forms of $S O(12, \mathbb{C})$ we get some other elements:

$\begin{array}{lllllll} & \mathrm{SO}(12) & \mathrm{SO}(10,2) & \mathrm{SO} *(12) & \mathrm{SO} *(12) & \mathrm{SO}(8,4) & \mathrm{SO}(6,6) \\ \mathrm{SO}(12) & 0 & 0 & 0 & 0 & 0 & 1 \\ \mathrm{SO}(10,2) & 0 & 0 & 0 & 0 & 15 & 66 \\ \mathrm{SO} *(12) & 0 & 0 & 0 & 60 & 0 & 692 \\ \mathrm{SO} *(12) & 0 & 0 & 60 & 0 & 0 & 692 \\ \mathrm{SO}(8,4) & 0 & 15 & 0 & 0 & 300 & 885 \\ \mathrm{SO}(6,6) & 1 & 66 & 692 & 692 & 885 & 2320\end{array}$

Note that this diagram is symmetric: $S O(12, \mathbb{C}$ ) is self-dual (and the equal rank and split inner classes coincide). For a discussion of the two versions of $S O^{*}(12)$ see Example 3.3. Note that the two versions of $S O^{*}(12)$ on the dual side account for the fact that $S O(6,6)$ has two distinct blocks of size 692 .

Example 8.9 We break the symmetry between $G$ and $G^{\vee}$ of the previous example by taking $\operatorname{Spin}(12, \mathbb{C})$, which is dual to $P S O(12, \mathbb{C})$.

$\begin{array}{lllllll} & \text { PSO (12) } & \text { PSO }(10,2) & \text { PSO* (12) } & \text { PSO } *(12) & \text { PSO }(8,4) & \text { PSO }(6,6) \\ \text { Spin(12) } & 0 & 0 & 0 & 0 & 0 & 1 \\ \text { Spin(10,2) } & 0 & 0 & 0 & 0 & 15 & 87 \\ \operatorname{Spin} *(12) & 0 & 0 & 0 & 60 & 0 & 692 \\ \operatorname{Spin} *(12) & 0 & 0 & 60 & 0 & 0 & 692 \\ \operatorname{Spin}(8,4) & 0 & 15 & 0 & 0 & 300 & 915 \\ \operatorname{Spin}(6,6) & 1 & 66 & 436 & 436 & 885 & 2180\end{array}$

There is an interesting phenomenon here. Note that $\operatorname{Spin}(6,6)$ has a block $\mathcal{B}_{\text {Spin }}$ of size 66 , dual to a block of $P S O(10,2)$. From the previous example $S O(6,6)$ also has a block of size 66 , denoted $\mathcal{B}_{S O}$, dual to a block for $S O(10,2)$. From the output of the block command one can see these blocks are isomorphic.

All representations in a block have the same central character, and the representations in $\mathcal{B}_{\text {Spin }}$ factor to the image of $\operatorname{Spin}(6,6)$ in $S O(6,6)$. The image of this map has index 2 in $S O(6,6)$. Therefore it is not obvious that $\mathcal{B}_{\text {Spin }}$ and $\mathcal{B}_{S O}$ should be isomorphic (and this doesn't happen for the blocks of $\operatorname{Spin}(6,6)$ of size 436 and 2,180).

The explanation is seen by looking at the dual side. The map $S O(10,2) \rightarrow$ $P S O(10,2)$ is surjective. It follows that these dual blocks are isomorphic, hence the blocks themselves are isomorphic. 
We leave it to the reader to see that the symmetry between the two versions of $S O^{*}(12)$ is broken by taking the "non-diagonal" quotient of $S O(12, \mathbb{C})$. See Examples 2.7 and 3.3.

Example 8.10 Here is the output of blocksizes for $E_{8}$, with the real forms added:

$\begin{array}{llll} & \text { compact } & \text { quaternionic } & \text { split } \\ \text { compact } & 0 & 0 & 1 \\ \text { quaternionic } & 0 & 3150 & 73410 \\ \text { split } & 1 & 73410 & 453060\end{array}$

The quaternionic real form is the one denoted e8(e7.su(2)) in the software. The software computes the structure of the block of size 453, 060 easily. However computing Kazhdan-Lusztig-Vogan polynomials for this block required special techniques. See www.liegroups.org for more information.

We now look at individual representations in a few examples. The cases of real forms of $S L(2, \mathbb{C})$ and $P S L(2, \mathbb{C})$ are explained in detail in [1, Example 12.20]. In particular see the table at the end of Section 12. Here is a brief summary.

Example 8.11 $S L(2, \mathbb{R})$ and $S U(2)$ :

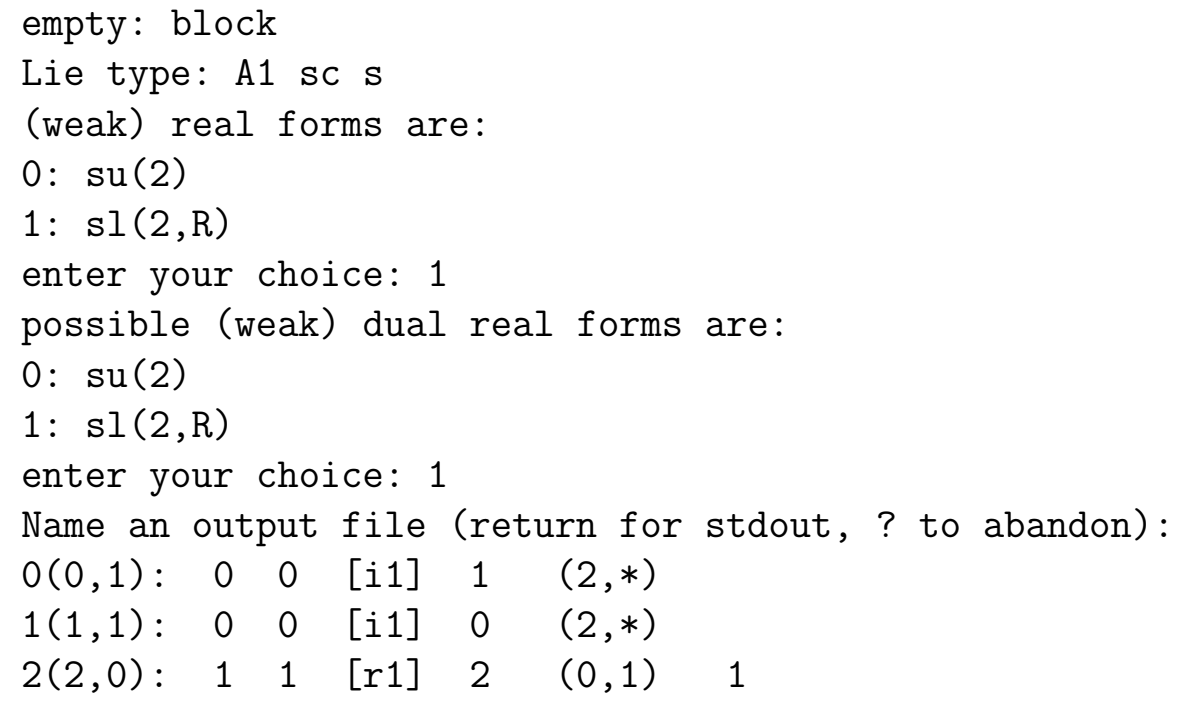


This is the block of $S L(2, \mathbb{R})$ of size 3 of example 8.5. We can take the infinitesimal character to be $\rho$, and this block contains the two discrete series representations \#0,\#1, and the trivial representation \#2. The singleton block of $S L(2, \mathbb{R})$ is found taking the dual group to be compact:

possible (weak) dual real forms are:

$0: \mathrm{su}(2)$

1: $\operatorname{sl}(2, R)$

enter your choice: 0

Name an output file (return for stdout, ? to abandon):

$0(2,0): 111 \quad[\mathrm{rn}] \quad 0 \quad(*, *) \quad 1$

This is an irreducible (non-spherical) principal series representation of $S L(2, \mathbb{R})$ at $\rho$ (with odd $K$-types).

Example 8.12 The adjoint group $P S L(2, \mathbb{R}) \simeq S O(2,1)$ has a block of size 3 dual to the preceding one:

$0(0,2): 00 \quad 0 \quad[i 2] \quad 0 \quad(1,2)$

$1(1,0): 1 \quad 1 \quad[r 2] \quad 2 \quad(0, *) \quad 1$

$2(1,1): \quad 1 \quad 1 \quad[r 2] \quad 1 \quad(0, *) \quad 1$

We can take infinitesimal character $\rho$, and the block consists of the unique discrete series representation $\# 0$ and the two one-dimensional representations $\# 1$,\#2. These are all of the irreducible representations of $\operatorname{PSL}(2, \mathbb{R})$ with infinitesimal character $\rho$.

As before taking the dual group to be compact we obtain a singleton:

possible (weak) dual real forms are:

$0: \mathrm{su}(2)$

1: $\operatorname{sl}(2, R)$

enter your choice: 0

Name an output file (return for stdout, ? to abandon):

$\begin{array}{lllllll}0(1,0): & 1 & 1 & {[\mathrm{rn}]} & 0 & (*, *) & 1\end{array}$

This block occurs at infinitesimal character $2 \rho$; in this case the set $\Lambda$ of Theorem 8.3 can be taken to be $\{\rho, 2 \rho\}$. Note that $S O(2,1)$ has two irreducible representation $P S_{ \pm}$at infinitesimal character $2 \rho$. The fact that there are two such representations (each of which is a block) is reflected in 
the fact that on the dual side there are two strong real forms $S U(2,0$ and $S U(0,2)$. See Examples 8.5 and 8.6, and the table at the end of $[1$, Section $12]$.

We illustrate the other information in the output of block by looking at $S p(4, \mathbb{R})$. See [1, Example 14.19].

Example 8.13 Here is the block of size 12 for $S p(4, \mathbb{R})$ :

$\begin{array}{rllllllll}0(0,6): & 0 & 0 & {[\mathrm{i} 1, \mathrm{i} 1]} & 1 & 2 & (6, *) & (4, *) & \\ 1(1,6): & 0 & 0 & {[\mathrm{i} 1, \mathrm{i} 1]} & 0 & 3 & (6, *) & (5, *) & (5,2) \\ 2(2,6): & 0 & 0 & {[\mathrm{ic}, \mathrm{i} 1]} & 2 & 0 & (*, *) & (4, *) & \\ 3(3,6): & 0 & 0 & {[\mathrm{ic}, \mathrm{i} 1]} & 3 & 1 & (*, *) & (5, *) & \\ 4(4,4): & 1 & 2 & {[\mathrm{C}+, \mathrm{r} 1]} & 8 & 4 & (*, *) & (0,2) & 2 \\ 5(5,4): & 1 & 2 & {[\mathrm{C}+, \mathrm{r} 1]} & 9 & 5 & (*, *) & (1,3) & 2 \\ 6(6,5): & 1 & 1 & {[\mathrm{r} 1, \mathrm{C}+]} & 6 & 7 & (0,1) & (*, *) & 1 \\ 7(7,2): & 2 & 1 & {[\mathrm{i} 2, \mathrm{C}-]} & 7 & 6 & (10,11) & (*, *) & 2,1,2 \\ 8(8,3): & 2 & 2 & {[\mathrm{C}-, i 1]} & 4 & 9 & (*, *) & (10, *) & 1,2,1 \\ 9(9,3): & 2 & 2 & {[\mathrm{C}-, i 1]} & 5 & 8 & (*, *) & (10, *) & 1,2,1 \\ 10(10,0): & 3 & 3 & {[\mathrm{r} 2, \mathrm{r} 1]} & 11 & 10 & (7, *) & (8,9) & 1,2,1,2 \\ 11(10,1): & 3 & 3 & {[\mathrm{r} 2, \mathrm{rn}]} & 10 & 11 & (7, *) & (*, *) & 1,2,1,2\end{array}$

The 12 representations, numbered $0, \ldots, 11$, are parametrized by pairs $(x, y)$, the second entry on each line, from the corresponding $\mathrm{kgb}$ commands for $G$ and $G^{\vee}$. See Example 7.6; note that for $G=S p(4, \mathbb{R})$ there are 11 orbits numbered $0, \ldots, 11$ and for $G^{\vee}=S O(3,2)$ there are 7 orbits, labelled $0, \ldots, 6$.

The next two columns give the Cartan subgroup, and length of the parameter, just as in the output of kgb for $x$ (Section 7).

The term in brackets list each of the simple roots as:

- compact imaginary: ic

- noncompact imaginary, type I/II: i1/i2

- complex: $\mathrm{C}+, \mathrm{C}-$

- real, not satisfying the parity condition: rn

- real, satisfying the parity condition type I/II: r1/r2 
For information about type I/II roots and the parity condition see [3, Section 8.3].

The next two columns give cross actions of the simple roots, similar to the kgb command, followed by two columns for Cayley transforms. These follow from the cross action/Cayley transforms for $\mathrm{kgb}$ on both the $G$ and $G^{\vee}$ side. In this setting (unlike kgb) these can be double valued, even for a noncompact imaginary root, since this corresponds to a real root on the dual side.

The final column is the corresponding twisted involution, exactly as in the kgb command for $x$.

Example 8.14 We conclude with a large example.

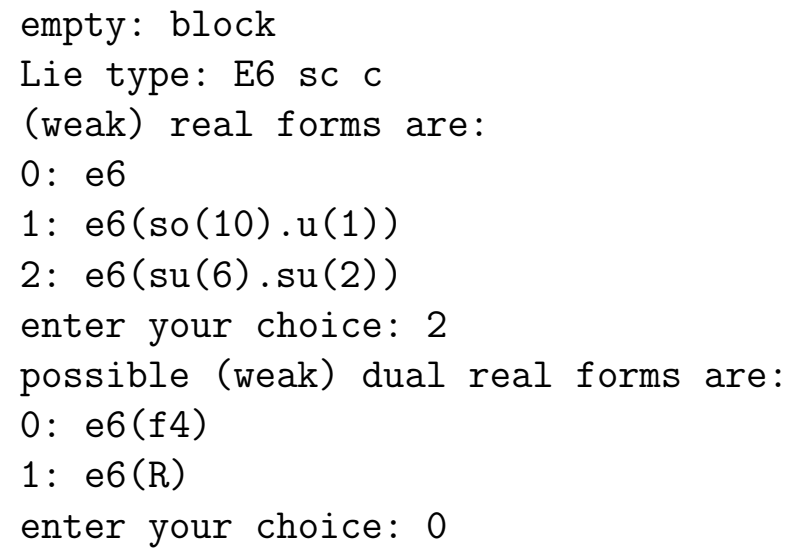

This is the block for $E_{6}\left(A_{5} \times A_{1}\right)$ (simply connected), dual to $E_{6}\left(F_{4}\right)$ (adjoint).

$\begin{array}{rrrlrrrrrr}0(851,44): & 8 & 4 & {[\mathrm{C}+, \mathrm{rn}, \mathrm{rn}, \mathrm{rn}, \mathrm{rn}, \mathrm{C}+]} & 2 & 0 & 0 & 0 & 0 & 1 \\ 1(1013,43): & 9 & 4 & {[\mathrm{C}+, \mathrm{rn}, \mathrm{rn}, \mathrm{rn}, \mathrm{C}+, \mathrm{C}-]} & 4 & 1 & 1 & 1 & 3 & 0 \\ 2(1014,42): & 9 & 4 & {[\mathrm{C}-, \mathrm{rn}, \mathrm{C}+, \mathrm{rn}, \mathrm{rn}, \mathrm{C}+]} & 0 & 2 & 5 & 2 & 2 & 4 \\ 3(1165,41): & 10 & 4 & {[\mathrm{C}+, \mathrm{rn}, \mathrm{rn}, \mathrm{C}+, \mathrm{C}-, \mathrm{rn}]} & 8 & 3 & 3 & 6 & 1 & 3 \\ 4(1166,40): & 10 & 4 & {[\mathrm{C}-, \mathrm{rn}, \mathrm{C}+, \mathrm{rn}, \mathrm{C}+, \mathrm{C}-]} & 1 & 4 & 9 & 4 & 8 & 2 \\ 5(1167,39): & 10 & 4 & {[\mathrm{rn}, \mathrm{rn}, \mathrm{C}-, \mathrm{C}+, \mathrm{rn}, \mathrm{C}+]} & 5 & 5 & 2 & 7 & 5 & 9 \\ 6(1304,38): & 11 & 4 & {[\mathrm{C}+, \mathrm{C}+, \mathrm{C}+, \mathrm{C}-, \mathrm{rn}, \mathrm{rn}]} & 11 & 10 & 10 & 3 & 6 & 6 \\ 7(1305,37): & 11 & 4 & {[\mathrm{rn}, \mathrm{C}+, \mathrm{rn}, \mathrm{C}-, \mathrm{C}+, \mathrm{C}+]} & 7 & 14 & 7 & 5 & 14 & 12 \\ 8(1306,36): & 11 & 4 & {[\mathrm{C}-, \mathrm{rn}, \mathrm{C}+, \mathrm{C}+, \mathrm{C}-, \mathrm{rn}]} & 3 & 8 & 13 & 11 & 4 & 8 \\ 9(1307,35): & 11 & 4 & {[\mathrm{rn}, \mathrm{rn}, \mathrm{C}-, \mathrm{C}+, \mathrm{C}+, \mathrm{C}-]} & 9 & 9 & 4 & 12 & 13 & 5 \\ 10(1430,34): & 12 & 4 & {[\mathrm{C}+, \mathrm{C}-, \mathrm{C}-, \mathrm{rn}, \mathrm{rn}, \mathrm{rn}]} & 18 & 6 & 6 & 10 & 10 & 10 \\ 11(1431,33): & 12 & 4 & {[\mathrm{C}-, \mathrm{C}+, \mathrm{C}+, \mathrm{C}-, \mathrm{rn}, \mathrm{rn}]} & 6 & 18 & 15 & 8 & 11 & 11 \\ 12(1432,32): & 12 & 4 & {[\mathrm{rn}, \mathrm{C}+, \mathrm{rn}, \mathrm{C}-, \mathrm{C}+, \mathrm{C}-]} & 12 & 19 & 12 & 9 & 17 & 7 \\ 13(1433,31): & 12 & 4 & {[\mathrm{rn}, \mathrm{rn}, \mathrm{C}-, \mathrm{C}+, \mathrm{C}-, \mathrm{rn}]} & 13 & 13 & 8 & 16 & 9 & 13 \\ 14(1434,30): & 12 & 4 & {[\mathrm{rn}, \mathrm{C}-, \mathrm{rn}, \mathrm{rn}, \mathrm{C}-, \mathrm{C}+]} & 14 & 7 & 14 & 14 & 7 & 19 \\ 15(1536,29): & 13 & 4 & {[\mathrm{C}+, \mathrm{C}+, \mathrm{C}-, \mathrm{C}+, \mathrm{rn}, \mathrm{rn}]} & 24 & 24 & 11 & 20 & 15 & 15\end{array}$




\begin{tabular}{|c|c|c|c|c|c|c|c|c|c|}
\hline & 3 & 4 & {$[\mathrm{rn}, \mathrm{C}+, \mathrm{C}+, \mathrm{C}-, \mathrm{C}+, \mathrm{rn}]$} & 6 & 2 & 20 & 3 & & \\
\hline 8,27$):$ & 13 & 2 & $\mathrm{rn}, \mathrm{C}+, \mathrm{rn}, \mathrm{C}+, \mathrm{C}-, \mathrm{C}+]$ & 7 & 3 & 7 & & & \\
\hline 39,26$):$ & 13 & 4 & $\mathrm{C}-, \mathrm{C}-, \mathrm{C}+, \mathrm{rn}, \mathrm{rn}, \mathrm{rn}]$ & & & 4 & 8 & & \\
\hline $9(1540,25):$ & 3 & & $\mathrm{rn}, \mathrm{C}-, \mathrm{rn}, \mathrm{rn}, \mathrm{C}+, \mathrm{C}-]$ & 9 & 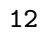 & 9 & 9 & & \\
\hline & 4 & & $+, \mathrm{C}+, \mathrm{C}-, \mathrm{C}-, \mathrm{C}+, \mathrm{rn}]$ & 8 & & 6 & 5 & & \\
\hline 21,23$)$ & 4 & & $\mathrm{cn}, \mathrm{C}+, \mathrm{C}+, \mathrm{C}-, \mathrm{C}-, \mathrm{C}+]$ & 21 & 9 & 25 & 17 & 6 & 27 \\
\hline$(1622,22)$ & 4 & & $\mathrm{n}, \mathrm{C}-, \mathrm{C}+, \mathrm{rn}, \mathrm{C}+, \mathrm{rn}]$ & 22 & 6 & 26 & 22 & 9 & \\
\hline & 4 & & $\mathrm{C}-, \mathrm{rn}, \mathrm{C}+, \mathrm{C}-, \mathrm{C}-$ & 23 & 7 & 23 & 27 & 9 & 17 \\
\hline 24 & 14 & & $\mathrm{C}-, \mathrm{C}-, \mathrm{C}+, \mathrm{rn}, \mathrm{rn}]$ & 5 & 5 & 18 & 8 & & \\
\hline 34 & 5 & & $, \mathrm{C}+, \mathrm{C}-, \mathrm{rn}, \mathrm{C}-, \mathrm{C}+]$ & 32 & 1 & 21 & 5 & 0 & $\mathrm{C}$ \\
\hline & 5 & & $, \mathrm{C}-, \mathrm{C}-, \mathrm{C}+, \mathrm{C}+, \mathrm{rn}]$ & 34 & 0 & 22 & 34 & 1 & \\
\hline & 15 & &,$+ \mathrm{C}+, \mathrm{C}-$ & 7 & 3 & 0 & 3 & & \\
\hline 87 & 15 & & $\mathrm{C}+, \mathrm{rn}, \mathrm{C}-, \mathrm{C}+, \mathrm{rn}]$ & 20 & 34 & 28 & 4 & 32 & ? \\
\hline 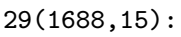 & 15 & & $\mathrm{C}-, \mathrm{C}+, \mathrm{C}+, \mathrm{C}-, \mathrm{C}$ & 29 & 21 & 31 & 33 & 2 & \\
\hline & 6 & & & & & & & & \\
\hline 1, & 16 & &, $\mathrm{C}-, \mathrm{C}-, \mathrm{C}+, \mathrm{C}-$, & 38 & & 29 & 35 & 26 & 36 \\
\hline 32.1 & 16 & & $, \mathrm{C}+, \mathrm{rn}, \mathrm{rn}, \mathrm{C}-, \mathrm{C}+]$ & 25 & 8 & 32 & 32 & 8 & 37 \\
\hline & 16 & & & & & & & & \\
\hline 4,10 & 16 & & $, \mathrm{C}-, \mathrm{rn}, \mathrm{C}-, \mathrm{C}+, \mathrm{rn}]$ & 26 & & 34 & & 38 & 34 \\
\hline 60,9 & 17 & & $, \mathrm{rn}, \mathrm{C}+, \mathrm{C}-, \mathrm{C}+, \mathrm{C}+]$ & 40 & 35 & 39 & 31 & 40 & 39 \\
\hline $6(1761,8)$ & & &, $\mathrm{C}-, \mathrm{C}-, \mathrm{C}+, \mathrm{rn}$ & & & & & & 31 \\
\hline $7(1762,7)$ & 17 & & $-, \mathrm{C}+, \mathrm{rn}, \mathrm{rn}, \mathrm{rn}, \mathrm{C}-]$ & 30 & 41 & 37 & 37 & 37 & 32 \\
\hline $8(1763,6)$ & 17 & & $-, \mathrm{C}-, \mathrm{rn}, \mathrm{C}+, \mathrm{C}-, \mathrm{C}+]$ & 31 & 32 & 38 & 10 & 34 & - \\
\hline $9(1777,5)$ & 18 & &,$- \mathrm{C}+, \mathrm{C}-$ & 3 & 39 & 5 & 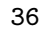 & 2 & \\
\hline $0(1778,4)$ & 18 & & $-, \mathrm{rn}, \mathrm{C}+, \mathrm{C}-, \mathrm{C}-, \mathrm{C}+]$ & 35 & & 42 & & 5 & \\
\hline $1(1779,3)$ & 18 & & $\mathrm{C}-, \mathrm{C}-, \mathrm{rn}, \mathrm{C}+, \mathrm{rn}, \mathrm{C}-]$ & 36 & 37 & 41 & 3 & 41 & . \\
\hline $2(1786,2)$ & 19 & & $, r n, C-$, & 44 & & 40 & & 39 & 44 \\
\hline $3(1787,1)$ & 19 & & $\mathrm{C}-, \mathrm{rn}, \mathrm{C}+, \mathrm{C}-, \mathrm{C}+, \mathrm{C}-]$ & 39 & & 44 & 41 & 44 & 40 \\
\hline $4(1790,0)$ & 20 & & {$[\mathrm{C}-, \mathrm{rn}, \mathrm{C}-, \mathrm{rn}, \mathrm{C}-, \mathrm{C}-]$} & 42 & 44 & 43 & 44 & 43 & \\
\hline
\end{tabular}

The last column (twisted involution) has been deleted, as have the Cayley transoforms, which are all empty, i.e. $(*, *)$.

All of these representations come from a the same Cartan subgroup. This is the entry 4 in each row; by the output of the cartan command this is the most split Cartan subgroup, and is isomorphic to $\mathbb{R}^{\times} \times \mathbb{R}^{\times} \times \mathbb{C}^{\times} \times \mathbb{C}^{\times}$. Note that every root is of type $\mathbf{C} \pm$ or $\mathrm{rn}$, so there are no Cayley transforms. This is surprising, since this group has 5 conjugacy classes of Cartan subgroups including a compact Cartan subgroup but not a split one. The reason is that the dual block is for the adjoint group $E_{6}\left(F_{4}\right)$, which has only one conjugacy class of Cartan subgroup (cf. Example 5.4).

There are 1,791 orbits of $K$ on $G / B$, and 45 for the dual group, given by the $\mathrm{kgb}$ command. In the parameters $(x, y)$ given in parentheses each $0 \leq y \leq 44$ appears once, since the dual Cartan subgroup is connected. The fact that 45 distinct values of $x$ (between 0 to 1790) occur is more subtle. 


\section{The Geometry of $\mathcal{X}^{r}$}

Fix $(G, \gamma)$ and let $\mathcal{X}^{r}$ be the reduced parameter space defined in Section 4. It is helpful to give some detail about the structure of $\mathcal{X}^{r}$. Recall (Section 4) there is a map from $\mathcal{X}^{r}$ to the space $\mathcal{I}_{W}$ of twisted involutions in $W$. We discuss the fibers of this map.

\subsection{The Adjoint Case}

Fix $(G, \gamma)$ with $G$ adjoint. This case is simplest, since real forms and strong real forms coincide. Fix $\tau \in \mathcal{I}_{W}$.

Let $H^{-\tau}=\left\{h \in H \mid \tau(h)=h^{-1}\right\}$. There is a natural simply transitive action of $H^{-\tau} /\left(H^{-\tau}\right)^{0}$ on $\mathcal{X}_{\tau}^{r}$ [1, Section 11]. Note that $H^{-\tau} /\left(H^{-\tau}\right)^{0} \simeq \mathbb{Z}_{2}^{b}$ where $b$ is the number of $S^{1}$ factors of the real form of $H$ defined by $\tau$. Fix $x \in \mathcal{X}_{\tau}^{r}$. Via a choice of basepoint in $\mathcal{X}_{\tau}^{r}$ the output of cartan identifies $\mathcal{X}_{\tau}^{r}$ with $\left\{0,1, \ldots, 2^{b}-1\right\}$. The element of $\mathbb{Z}_{2}^{b}$ corresponding to $0 \leq k \leq 2^{b}-1$ is the binary expansion of $k$.

The rank of $\mathcal{X}_{\tau}^{r}$ is also given by fiber rank in the output of cartan. For each Cartan the number \#X_r is the number of strong involutions lying over this conjugacy class in $\mathcal{I}_{W}$, i.e. twisted involution orbit size $\times 2 \wedge$ fiber rank.

The imaginary Weyl group $W_{i, \tau}$ (the Weyl group of the imaginary roots with respect to $\tau$ ) acts on $\mathcal{X}_{\tau}^{r}$, and the orbits are in one to one correspondence with real forms containing this Cartan subgroup. The cartan command gives this decomposition. For example a line

real form \#2: $[0,1,2]$ (3)

means that corresponding to real form \#2 (from the output of the realform command) are three elements of $\mathcal{X}_{\tau}^{r}$, labelled 0,1,2 (the number in parentheses is the number of elements in brackets).

Example 9.1 Here are the equal rank real forms of $P S L(4, \mathbb{C})$ :

empty: cartan

Lie type: A3 ad c

(weak) real forms are:

$0: \mathrm{su}(4)$

1: $\operatorname{su}(3,1)$

2: $\operatorname{su}(2,2)$ 
enter your choice: 2

Name an output file (return for stdout, ? to abandon):

Cartan \#0:

split: 0 ; compact: 3 ; complex: 0

canonical twisted involution:

twisted involution orbit size: 1; fiber rank: 3; \#X_r: 8

imaginary root system: A3

real root system is empty

complex factor is empty

real form \#2: $[0,2,5]$ (3)

real form \#1: $[1,3,4,6]$ (4)

real form \#0: [7] (1)

This is the fundamental fiber $\tau=\delta$. In this case $W_{i, \delta}=W$. This fiber has $2^{3}=8$ elements. There is a fixed point of the action of $W$ on $\mathcal{X}_{\delta}$, corresponding to the compact real form $P S U(4)$ (realform \#0). The largest orbit, of size 4 , corresponds to the group $S U(3,1)$.

Here are the remaining Cartan subgroups:

Cartan \#1:

split: 0 ; compact: 1 ; complex: 1

canonical twisted involution: $1,2,3,2,1$

twisted involution orbit size: 6; fiber rank: 1; \#X_r: 12

imaginary root system: A1

real root system: $\mathrm{A} 1$

complex factor is empty

real form \#2: [0] (1)

real form \#1: [1] (1)

Cartan \#2:

split: 1 ; compact: 0 ; complex: 1

canonical twisted involution: $2,1,3,2$

twisted involution orbit size: 3 ; fiber rank: 0; \#X_r: 3

imaginary root system is empty

real root system: A1.A1

complex factor: A1

real form \#2: [0] (1) 
There are 6 elements $\tau$ here for which $\mathcal{X}_{\tau}^{r}$ has order 2; both noncompact real forms appear. Finally only the quasisplit group $P S U(3,3)$ contains the maximally split Cartan, in which case there are 3 twisted involutions $\tau$, each with $\left|\mathcal{X}_{\tau}^{r}\right|=1$

Therefore $\mathcal{X}^{r}$ has $1 \times 8+6 \times 2+3 \times 1=23$ elements. See Example 7.5

\subsection{Strong Real Forms and Fibers}

The strongreal command gives information about strong real forms (see Section 4), which may also be interpreted as information about the fibers $p: \mathcal{X}^{r} \rightarrow \mathcal{I}_{W}$ in the general case.

The strong real forms "containing" $(H, \tau)$ are parametrized by $\mathcal{X}_{\tau}^{r} / W_{i, \tau}$. In particular every strong real form contains the fundamental Cartan subgroup $(H, \delta)$, so strong real forms are parametrized by $\mathcal{X}_{\delta}^{r} / W_{i, \delta}$. Things are particularly simple if $G$ has equal rank, say $n$, in which case

$$
\mathcal{X}_{\delta}^{r} / W \simeq\left\{h \in H \mid h^{2} \in Z^{r}\right\} / W .
$$

Note that $\left|\mathcal{X}_{\delta}^{r}\right|=\left|Z^{r}\right| 2^{n}$ in this case.

In general not every element of $Z^{r}$ is of the form $x^{2}$ for some $x \in \mathcal{X}$. The software labels the elements which are of this form as $\left\{z_{0}, \ldots, z_{r-1}\right\}$, denoted class \#0, class \#1,..class \#r-1. In fact $r=2^{s}$ for some $s$. For each $z_{j}$ the set $\mathcal{X}_{\tau}^{r}\left(z_{j}\right)=\left\{x \in \mathcal{X}_{\tau} \mid x^{2}=z_{j}\right\}$ is isomorphic to $H^{-\tau} /\left(H^{-\tau}\right)^{0}$ (independent of $j$ ) which has cardinality $2^{b}\left(b\right.$ is the number of $S^{1}$ factors as in the preceding section) so $\left|\mathcal{X}_{\tau}^{r}\right|=2^{b+s}$.

The group $W_{i, \tau}$ acts on $\mathcal{X}_{\tau}\left(z_{j}\right)$, and the orbits correspond to strong real forms. All of this information is given by the strongreal command. See [1, Section 11] and the help file for the strongreal command.

Note that the order of $\mathcal{X}^{r}$ may be computed by summing over Cartan subgroups, taking into account the size of the conjugacy classes of twisted involutions.

Example 9.3 Consider the equal rank inner class of $S L(4, \mathbb{C})$. Compare Example 9.1.

main: strongreal

Lie type: A3 sc C

(weak) real forms are:

$0: \mathrm{su}(4)$ 


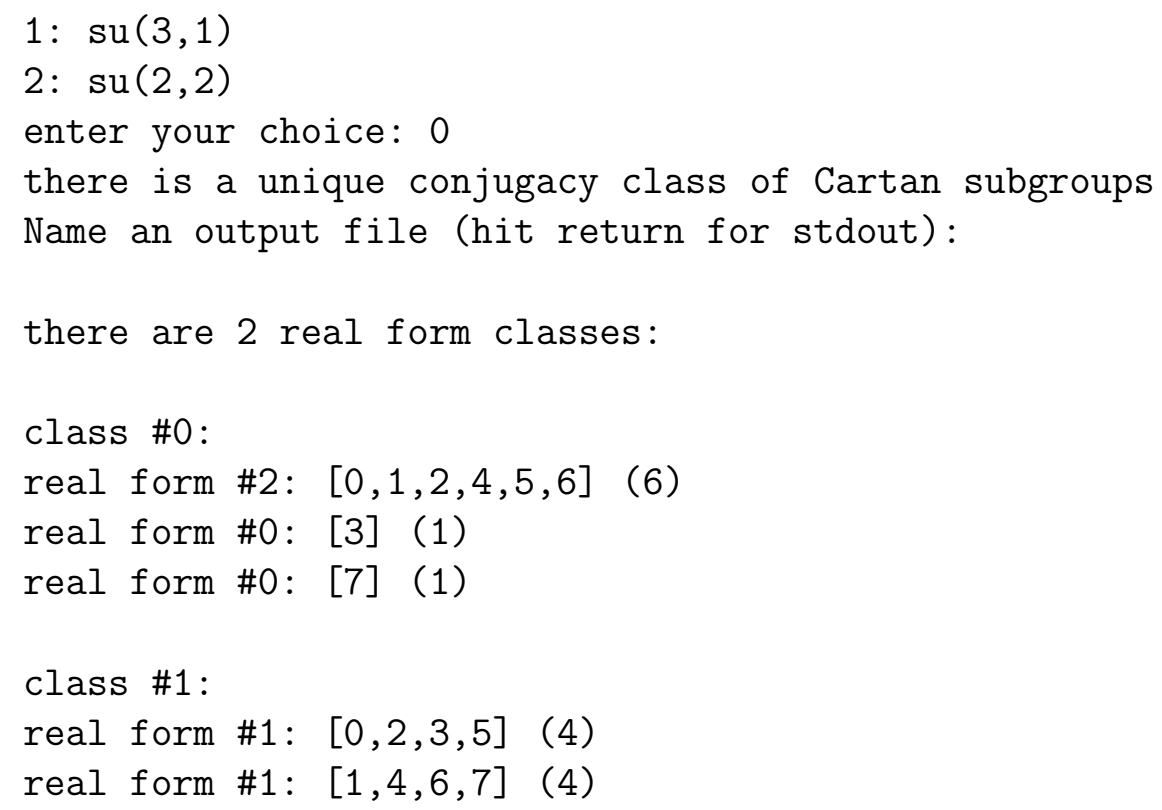

First of all, reading the lines beginning realform, we see there is one strong real form mapping to $S U(2,2)$ (real form \#2), two mapping to $S U(4)$ (real form \#0), and two mapping to $S U(3,1)$ (real form \#1). We can think of these as $S U(4,0), S U(3,1), S U(2,2), S U(1,3)$, and $S U(0,4)$.

This is the fundamental fiber, i.e. $\tau=\delta$. In this case $Z \simeq \mathbb{Z}_{4}$ and $Z / Z^{2} \simeq \mathbb{Z}_{2}$, so $\left|Z^{r}\right|=2$. In this case $b$ (the number of $S^{1}$ factors) is 3 , so $\left|\mathcal{X}_{\delta}^{r}(z)\right|=8$ for each $z \in Z^{r}$ (it is never 0 in this case), and $\left|\mathcal{X}_{\delta}^{r}\right|=16$. We can take $Z / Z^{2}=\left\{z_{0}, z_{1}\right\}=\{I, i I\}$. These are the elements class \#0 and class \#1 respectively.

There are three orbits of $W=W_{i, \delta}$ on $\mathcal{X}_{\delta}^{r}(I)$. The $W$-orbit of $\operatorname{diag}(1,1,-1,-1)$ has 6 elements; given in the line real form \#2: $[0,1,2,4,5,6]$ (6); the corresponding real form is $S U(2,2)$. The elements $\pm I$ are each fixed by $W$, and correspond to the two lines beginning real form \#0. (The software does not specify which of these is $I$, and which $-I$ ).

We could have chosen $z_{0}=-I$ instead of $I$. Then $S U(2,2)$ would be given by $i$ times the preceding ones, i.e. the $W$-orbit of $\operatorname{diag}(i, i,-i,-i)$. Similarly the two strong real forms mapping to $S U(2)$ would be $\pm i I$. The software does not make an actual choice of $Z^{r}$; the output of the software, and the combinatorics of the algorithm, are independent of any such choice. See [1, Section 13].

Now consider $z=i I$. Then $\mathcal{X}_{\delta}^{r}(i I)$ consists of the elements $\zeta( \pm 1, \ldots, \pm 1)$ 
where $\zeta=e^{\pi i / 4}$, with an odd number of plus signs. The 8 such elements constitute $2 W$-orbits, hence the two strong real forms mapping to $S U(3,1)$.

Here are the strong real forms containing the other two Cartan subgroups in this example.

real: strongreal

cartan class (one of $0,1,2): 1$

Name an output file (return for stdout, ? to abandon):

there are 2 real form classes:

class \#0:

real form \#2: $[0,1]$ (2)

class \#1:

real form \#1: [0] (1)

real form \#1: [1] (1)

The compact real form real form \#0 only contains the compact Cartan subgroup and doesn't occur. In this case $\left|\mathcal{X}_{\tau}^{r}(I)\right|=\left|\mathcal{X}_{\tau}^{r}(i I)\right|=2,\left|\mathcal{X}_{\tau}^{r}\right|=4$, and both $S U(2,2)$ and $S U(3,1)$ occur.

real: strongreal

cartan class (one of $0,1,2): 2$

Name an output file (return for stdout, ? to abandon):

real form \#2: [0] (1)

For the most split Cartan subgroup $\left|\mathcal{X}_{\tau}^{r}(I)\right|=0,\left|\mathcal{X}_{\tau}^{r}(i I)\right|=1$, and only $S U(2,2)$ occurs.

The number of twisted involutions in each conjugacy class are 1,6 and 3, as given by the cartan command. Therefore the cardinality of $\mathcal{X}^{r}$ is

$$
16 \times 1+4 \times 6+1 \times 3=43 .
$$

See Example 7.3.

Example 9.5 Type $A_{2 n}$ is a little different than $A_{2 n+1}$. Here are the fundamental fiber and the equal rank strong real forms of $S L(5, \mathbb{C})$ : 
Lie type: A4 sc c

main: strongreal

(weak) real forms are:

$0: \mathrm{su}(5)$

1: $\operatorname{su}(4,1)$

2: $\operatorname{su}(3,2)$

enter your choice: 0

there is a unique conjugacy class of Cartan subgroups

Name an output file (hit return for stdout):

real form \#2: $[0,1,2,4,5,6,8,10,11,13]$ (10)

real form \#1: $[3,9,12,14,15]$ (5)

real form \#0: [7] (1)

In this case $Z \simeq \mathbb{Z} / 5 \mathbb{Z}$ and $Z / Z^{2}=1$. We can take $z=I$ in this case, and the map from strong real forms to real forms is bijective.

We conclude with a discussion of the inner class of $S L(n, \mathbb{R})$. If $n$ is odd this is the unique real form in this inner class; if $n$ is even there is one other real form $S L(n / 2, \mathbb{H})$. We can take $\theta\left(\operatorname{diag}\left(z_{1}, \ldots, z_{n}\right)\right)=\operatorname{diag}\left(\frac{1}{z_{n}}, \ldots, \frac{1}{z_{1}}\right)$, and $Z^{r}=\{I\}$ if $n$ is odd, or $\{ \pm I\}$ if $n$ is even.

Here is an example of each case.

Example 9.6 There is only one strong real form in the inner class of $S L(3, \mathbb{R})$ :

empty: strongreal

Lie type: A2 sc s

there is a unique real form: $s l(3, R)$

Here $Z^{r}=\{I\}$ and

$$
\mathcal{X}_{\delta}(I)=\left\{\operatorname{diag}(z, w, z) \mid z^{2} w=1\right\} /\left\{\operatorname{diag}\left(a c, b^{2}, a c\right) \mid a b c=1\right\}=I
$$

Example 9.8 Now consider the inner class of $S L(4, \mathbb{R})$, which also contains the real form $S L(2, \mathbb{H})$. In this case $Z= \pm I$.

empty: strongreal

Lie type: A5 sc s

(weak) real forms are:

$0: \operatorname{sl}(3, \mathrm{H})$ 


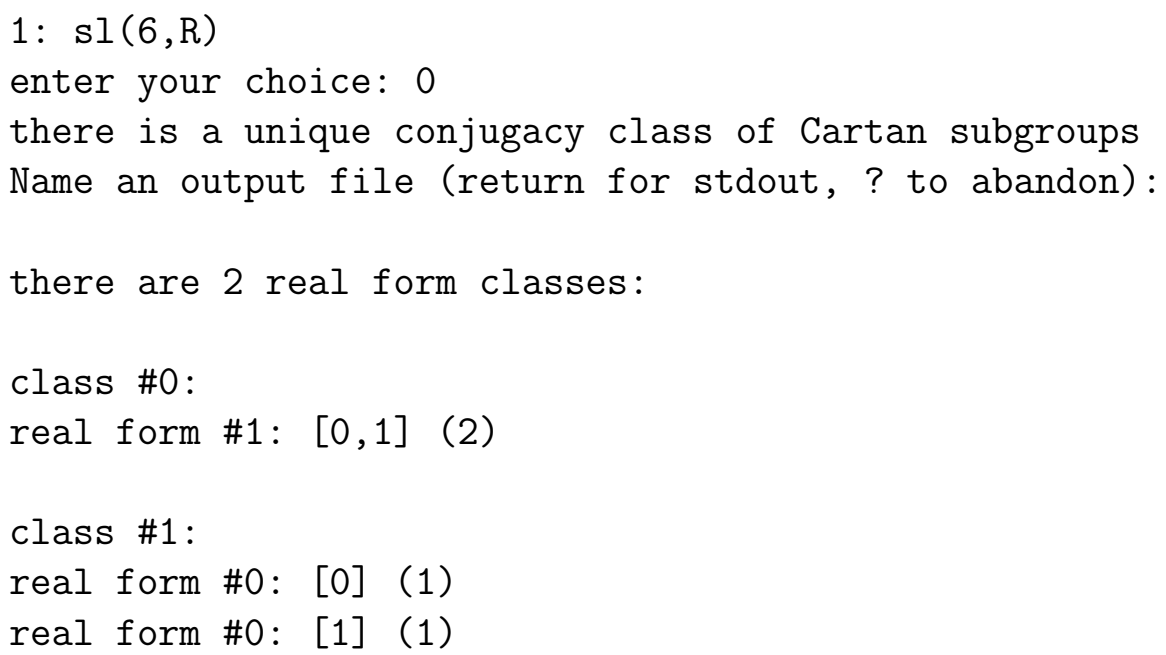

We compute

$$
\begin{aligned}
H^{-\tau} /\left(H^{-\tau}\right)^{0} & =\left\{\operatorname{diag}(z, w, w, z) \mid z^{2} w^{2}=1\right\} /\{\operatorname{diag}(a d, b c, b c, a d) \mid a b c d=1\} \\
& =\{I, \operatorname{diag}(1,-1,-1,1)\}
\end{aligned}
$$

Taking $z=I$ (this is class \#1) we therefore have

$$
\mathcal{X}_{\delta}(I)=\{\delta, \operatorname{diag}(1,-1,-1,1) \delta\}
$$

These elements are not conjugate by $W$, so there are two strong real forms mapping to the real form $S L(2, \mathbb{H})$ (real form \#0).

On the other hand take $z=-I$ (class \#0). Then $x=\operatorname{diag}(1,1,-1,-1) \delta \in$ $\mathcal{X}_{\delta}(-I)$, and $\mathcal{X}_{\delta}(-I)$ is obtained by multiplying $\mathrm{x}$ on the left by $H^{-\tau} /\left(H^{-\tau}\right)^{0}$. This gives

$$
\mathcal{X}_{\delta}(-I)=\{\operatorname{diag}(1,1,-1,-1) \delta, \operatorname{diag}(1,-1,1,-1) \delta\} .
$$

These elements are conjugate by $W$, so there is only one strong real form mapping to the real form $S L(4, \mathbb{R})$ (real form \#1).

\section{References}

[1] Jeffrey Adams and Fokko du Cloux. Algorithms for representation theory of real reductive groups, http://www.liegroups.org/papers. To appear in J. Inst. Math. Jussieu 
[2] Anthony Knapp. Representation Theory of Semisimple Groups. An overview based on Examples. Princeton University Press, Princeton, NJ, 1986.

[3] David A. Vogan, Jr. Representations of Real Reductive Lie Groups, volume 15 of Progress in mathematics. Birkhäuser, Boston, 1981.

[4] David A. Vogan, Jr. Irreducible characters of semisimple Lie groups IV. character-multiplicity duality. Duke Math. J., 49, No. 4:943-1073, 1982. 